\title{
Stabilization for Networked Control Systems with Random Sampling Periods
}

\author{
Yuan Li, ${ }^{1,2}$ Qingling Zhang, ${ }^{1}$ Shuanghong Zhang, ${ }^{1,3}$ and Min $\mathrm{Cai}^{4}$ \\ ${ }^{1}$ Institute of Systems Science, Northeastern University, Shenyang 110819, China \\ ${ }^{2}$ School of Science, Shenyang University of Technology, Shenyang 110870, China \\ ${ }^{3}$ School of Science, Jilin Normal University, Siping 136000, China \\ ${ }^{4}$ School of Science, Dalian Jiaotong University, Dalian 116028, China
}

Correspondence should be addressed to Qingling Zhang; qlzhang@mail.neu.edu.cn

Received 13 November 2012; Accepted 30 December 2012

Academic Editor: Reinaldo Martinez Palhares

Copyright (C) 2013 Yuan Li et al. This is an open access article distributed under the Creative Commons Attribution License, which permits unrestricted use, distribution, and reproduction in any medium, provided the original work is properly cited.

\begin{abstract}
This paper investigates the stabilization of networked control systems (NCSs) with random delays and random sampling periods. Sampling periods can randomly switch between three cases according to the high, low, and medium types of network load. The sensor-to-controller $(S-C)$ random delays and random sampling periods are modeled as Markov chains. The transition probabilities of Markov chains do not need to be completely known. A state feedback controller is designed via the iterative linear matrix inequality (LMI) approach. It is shown that the designed controller is two-mode dependent and depends on not only the current $S$-C delay but also the most recent available sampling period at the controller node. The resulting closed-loop systems are special discrete-time jump linear systems with two modes. The sufficient conditions for the stochastic stability are established. An example of the cart and inverted pendulum is given to illustrate the effectiveness of the theoretical result.
\end{abstract}

\section{Introduction}

Networked control systems (NCSs) are feedback control systems whose feedback paths are implemented by a realtime network. Recently, much attention has been paid to the study of NCSs, due to their low cost, reduced weight and power requirements, simple installation and maintenance, high reliability, and so on $[1,2]$.

A basic problem in NCSs is the stability of the systems. In real-time control systems, three main issues are bandwidth and packet size constraints, time delays, and packet losses, which will degrade the performance of control systems and even make systems unstable; so it is significant to overcome the adverse influences of time delays, packet losses, and dynamic bandwidth [3-7].

On the other hand, the Markov chain, a discretetime stochastic process with the Markov property, can be effectively used to model NCSs with random time delays and random sampling periods, which are modeled as the Markovian jump linear systems (MJLSs). Recently, there have been considerable research efforts into NCSs [8-12]. For example, Xiao et al. present an $V-K$ iteration algorithm to design stabilizing controllers for specially structured discretetime jump linear systems with random but bounded delays in the feedback loop [8]. Zhang et al. propose a promising twomode-dependent-state feedback scheme to stabilize section with the current $S$ - $C$ delay $\left(\tau_{k}\right)$ and the previous controllerto-actuator $(C-A)$ delay $\left(d_{k-1}\right)$ modeled as two Markov chains [9]. Shi and Yu investigate the output feedback stabilization and robust mixed $\mathrm{H}_{2} / \mathrm{H}_{\infty}$ control of NCSs, in which it is assumed that at each sampling instant, the current $S$ - $C$ delay $\left(\tau_{k}\right)$ and the most recent available $C$ - $A$ delay $\left(d_{k-1-\tau_{k}}\right)$ can be obtained $[10,11]$. Huang and Nguang discuss the stabilization problem for a class of linear uncertain continuous NCSs, in which it is assumed that random communication $S-C$ delays $(\tau(t))$ and random communication $C$-A delays $(\rho(t))$ are modeled two continuous time-discrete-state Markov processes [12].

In the above references, the transition probabilities are assumed completely accessible and considered as the 
available knowledge for analyzing and designing the NCSs. In practice, this kind of information including the variation of time delays and packet losses is hard to obtain. The problems of partly unknown transition probabilities were investigated [13-16]. Zhang et al. investigate the stability of Markovian jump linear systems with partly unknown transition probabilities $[13,14]$. Wang et al. study the partially mode-dependent $H_{\infty}$ filtering problem for discrete-time Markovian jump systems with partly unknown transition probabilities [15]. Sun and Qin investigate the stability and stabilization problems of a class of NCSs with bounded packet dropout, in which the transition probabilities are partly unknown due to the complexity of network [16]. However, the developed controllers of these references [13-16] only are either mode independent or one-mode dependent, and the design problem can thus be readily converted into a standard MJLS problem. To the best of the authors' knowledge, if the transition probabilities are assumed partly accessible, designing the two-mode-dependent controller that simultaneously depends on both the current $S$ - $C$ delay $\left(\tau_{k}^{s c}\right)$, and the most recent available sampling period $\left(h_{k-\tau_{k}^{s c}}\right)$ has not been fully investigated, which is the focus of this work. When considering both $\tau_{k}^{s c}$ and $h_{k-\tau_{k}^{s c}}$, the resulting closedloop system can be transformed to a special MJLS, and thus the well-developed results on MJLSs with partly unknown transition probabilities cannot be directly applied [13-16].

In this paper, the stochastic stability of NCSs with random time delays and random sampling periods is studied, in which time delays and sampling periods are driven by two finitestate Markov chains. This paper is organized as follows. In Section 2, the NCSs model with random $S$ - $C$ time delays and random sampling periods is made, which is equivalent to a class of special discrete-time jump linear systems with two modes. Sufficient and necessary conditions of stochastic stability with completely known transition probabilities for the foregoing model are considered in Section 3. Sufficient conditions of stochastic stability with partly unknown transition probabilities for the foregoing model are considered in Section 4. Section 5 is an illustrative example, and our work in this paper is summarized in Section 6.

Notation. In this paper, $\mathbf{R}$ is the set of all real numbers, $\mathbf{R}^{n}$ denotes the $n$-dimensional Euclidean space. $(\Omega, F, P)$ denotes the probability space. $\mathbf{A}^{T}$ and $\mathbf{A}^{-1}$ denote the transpose and the inverse of a matrix $\mathbf{A}$, respectively. $\mathbf{A}>\mathbf{0}(\mathbf{A}<\mathbf{0})$ means that $\mathbf{A}$ is positive definite (negative definite). $\mathbf{0}$ and $\mathbf{I}$ are the zero and identity matrices with appropriate dimensions, respectively. In symmetric block matrices, we use an asterisk $(*)$ to represent a term that is induced by symmetry.

\section{Problem Formulation}

The structure of the considered NCSs is shown in Figure 1, where the plant is described by the following linear system model:

$$
\dot{x}(t)=A x(t)+B u(t)
$$

where $\mathrm{x}(\mathrm{t}) \in \mathbf{R}^{n}, u(t) \in \mathbf{R}^{m}$ are the system state vector and control input vector, respectively; $A, B$ are known constant

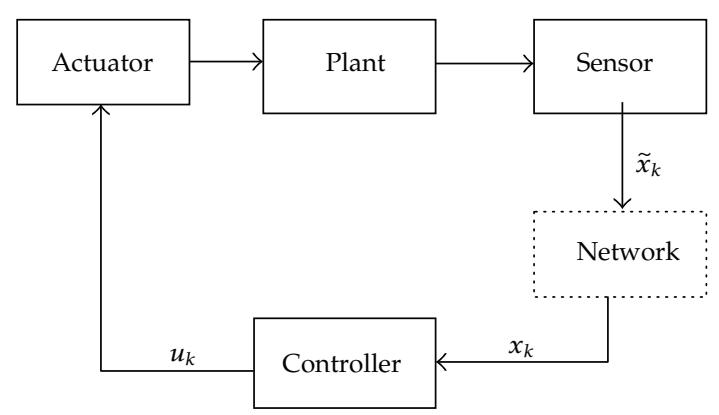

FiguRE 1: Diagram of NCSs.

matrices of appropriate dimensions. Suppose the sensor is clock-driven, the controller and the actuator are eventdriven.

Suppose bounded random delays only exist in the link from sensor to controller, as shown in Figure 1. Here, $0 \leq$ $\tau_{k}^{s c} \leq \tau^{s c}$ represents the $S$ - $C$ delay. The state feedback controller is to be designed.

For NCSs, the shorter the sampling period, the better system performance; however, the short sampling period will increase the possibility of network congestion. If the constant sampling period is adopted, the sampling period should be large enough to avoid network congestion, so network bandwidth cannot be sufficiently used when the network is idle. In [17-19], the variable sampling method is used, and the sampling periods are assumed to switch in a finite discrete set. But when the systems switch too fast, it is apt to cause oscillation and instability of the system. In the actual network, the size of sampling period is closely related to the network load. However, the network load usually is random [20]. As a result of the above, network load can be high load, low load, and medium load. Correspondingly, sampling periods can randomly switch between three cases of maximum, medium, and minimum in this paper. In the following, we consider that sample periods randomly switch between three cases and make NCSs model with random time delays and random sample periods. Thus, not only the network bandwidth can be fully used, but also the conservativeness of the stabilization conditions of NCSs can be reduced.

Suppose $h_{k}$ is the length of the $k$ th sampling period, if the network is idle, define the sampling period as $h_{\max }$, and if the network is occupied by the most users, define the sampling period as $h_{\min }$, otherwise define the sampling period as $(1 / 2)\left(h_{\max }+h_{\min }\right)$. Then, the sampling period $h_{k} \in$ $\left\{h_{\min },(1 / 2)\left(h_{\max }+h_{\min }\right), h_{\max }\right\}$.

The discrete-time expression of the system (1) is as follows:

$$
x(k+1)=\Phi\left(h_{k}\right) x(k)+\Gamma\left(h_{k}\right) u(k)
$$

where $\Phi\left(h_{k}\right)=e^{A h_{k}}$, and $\Gamma\left(h_{k}\right)=\int_{0}^{h_{k}} e^{A t} d t B$.

In this paper, $\tau_{k}^{s c}$ and $h_{k}$ are modeled as two homogeneous Markov chains defined in $(\Omega, F, P)$ that take values in $\varphi=\left\{0,1, \ldots, \tau^{s c}\right\}$ and $\phi=\{1,2,3\}$, and their transition 
probability matrices are $\Lambda=\left[\lambda_{i j}\right]$ and $\Pi=\left[\pi_{r s}\right]$, respectively, and

$$
\begin{aligned}
& \lambda_{i j}=\operatorname{Pr}\left\{\tau_{k+1}^{s c}=j \mid \tau_{k}^{s c}=i\right\}, \\
& \pi_{r s}=\operatorname{Pr}\left\{h_{k+1}=s \mid h_{k}=r\right\},
\end{aligned}
$$

where $\lambda_{i j}, \pi_{r s} \geq 0$, and $\sum_{j=0}^{\tau^{s c}} \lambda_{i j}=1, \sum_{s=1}^{3} \pi_{r s}=1$, for all $i, j \in \varphi$ and $r, s \in \phi$.

Remark 1. In this paper, we assume that the controller will always use the most recent data [8]. Thus, if we have $x\left(k-\tau_{k}^{s c}\right)$ at sampling instant $t_{k}$, then at sampling time $t_{k+1}$, even if there are delays longer than 1 or package loss, we still have $x\left(k-\tau_{k}^{s c}\right)$ to use. So in our model of the system in Figure 1 , the delay $\tau_{k}^{s c}$ can increase at most 1 each step, $\operatorname{Pr}\left\{\tau_{k+1}^{s c}>\tau_{k}^{s c}+1\right\}=0$.

In NCSs, to reduce the conservativeness of the stabilization conditions, it is desirable to consider not only the time delay but also the sample period information in the controller design. For the controller node, at time instant $t_{k}, \tau_{k}^{s c}$ can be obtained by comparing the current time and the time-stamp of the sensor information received. However, this sample period information cannot be received by the controller immediately because it needs to be transmitted through the network from sensor to controller. So if the time delay $\tau_{k}^{s c}$ exists, the information $h_{k-\tau_{k}^{s c}}$ of time instant $t_{k}$ would be known at the controller node. Consequently, it is desirable to design the state feedback controller that simultaneously depends on both $\tau_{k}^{s c}$ and $h_{k-\tau_{k}^{s c}}$. The mode-dependent state feedback control law is

$$
u(k)=K\left(\tau_{k}^{s c}, h_{k-\tau_{k}^{s c}}\right) \hat{x}(k)=K\left(\tau_{k}^{s c}, h_{k-\tau_{k}^{s c}}\right) x\left(k-\tau_{k}^{s c}\right),
$$

where $\widehat{x}(k)$ is the input vector of the state feedback controller, and $K$ is the controller gain to be designed.

In the following, we make the closed-loop system model. Substituting the formula (4) into (2), we can obtain the following closed-loop NCSs:

$$
x(k+1)=\Phi\left(h_{k}\right) x(k)+\Gamma\left(h_{k}\right) K\left(\tau_{k}^{s c}, h_{k-\tau_{k}^{s c}}\right) x\left(k-\tau_{k}^{s c}\right) .
$$

Augment the plant's state variable as

$$
X(k)=\left[\begin{array}{lllll}
x^{T}(k) & x^{T}(k-1) & \ldots & x^{T}\left(k-\tau^{s c}\right)
\end{array}\right]^{T},
$$

where $X(k) \in \mathbf{R}^{\left(\tau^{s c}+1\right) n}$, then we have

$$
\begin{aligned}
X(k+1) & =\left(\bar{A}\left(h_{k}\right)+\bar{B}\left(h_{k}\right) K\left(\tau_{k}^{s c}, h_{k-\tau_{k}^{s c}}\right) \bar{C}\left(\tau_{k}^{s c}\right)\right) X(k) \\
& =\widetilde{A}\left(\tau_{k}^{s c}, h_{k-\tau_{k}^{s c}}, h_{k}\right) X(k),
\end{aligned}
$$

where

$$
\begin{aligned}
& \bar{A}\left(h_{k}\right)=\left[\begin{array}{ccccc}
\Phi\left(h_{k}\right) & 0 & \cdots & 0 & 0 \\
I & 0 & \cdots & 0 & 0 \\
0 & I & \cdots & 0 & 0 \\
\vdots & \vdots & \ddots & \vdots & \vdots \\
0 & 0 & \cdots & I & 0
\end{array}\right], \\
& \bar{B}\left(h_{k}\right)=\left[\begin{array}{c}
\Gamma\left(h_{k}\right) \\
0 \\
0 \\
\vdots \\
0
\end{array}\right] \text {, }
\end{aligned}
$$

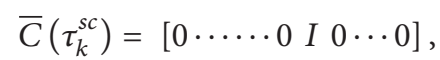

$$
\begin{aligned}
& \widetilde{A}\left(\tau_{k}^{s c}, h_{k-\tau_{k}^{s c}}, h_{k}\right)=\bar{A}\left(h_{k}\right)+\bar{B}\left(h_{k}\right) K\left(\tau_{k}^{s c}, h_{k-\tau_{k}^{s c}}\right) \bar{C}\left(\tau_{k}^{s c}\right) \text {, }
\end{aligned}
$$

and $\bar{C}\left(\tau_{k}^{s c}\right)$ has all elements being zero except for the $\tau_{k}^{s c}+1$ th block being an identity matrix.

Remark 2. Because the controller in (4) is two-mode dependent, the resulting closed-loop system in (7) depends on $\tau_{k}^{s c}, h_{k}, h_{k-\tau_{k}^{s c}}$ and can be transformed to a special MJLS. In addition, $h_{k-\tau_{k}^{s c}}$ is related to both $\tau_{k}^{s c}$ and $h_{k}$.

It can be seen that the closed-loop system in (7) is a jump linear system with two modes modeled by different homogeneous Markov chains. The objective of this paper is to design the state feedback controller (4) to guarantee the stochastic stability of the NCSs in (7). For the stochastic stability, we adopt the following definition [9].

Definition 3. The system in (7) is stochastically stable if for every finite matrix $X_{0}=X(0)$, initial mode $\tau_{0}^{s c}=\tau^{s c}(0) \in \varphi$, and $h_{-\tau_{0}^{s c}}=h\left(-\tau_{0}^{s c}\right) \in \phi$, there exists a finite matrix $W>0$ such that the following holds:

$$
\varepsilon\left\{\sum_{k=0}^{\infty}\|X(k)\|^{2} \mid X_{0}, \tau_{0}^{s c}, h_{-\tau_{0}^{s c}}\right\}<X_{0}^{T} W X_{0} .
$$

\section{The Case of Completely Known Transition Probabilities}

In this section, we first give the sufficient and necessary conditions for the state feedback stabilization of NCSs with completely known transition probabilities in (7) and then derive the equivalent conditions of LMIs with nonconvex constraints.

Because $h_{k-\tau_{k}^{s c}}$ is to be considered into the controller design, the multistep jump problem of Markov chains is involved in the system design. According to the ChapmanKolmogorov $(C-K)$ equation of stochastic process [21], we give the multistep transition probability as follows.

Lemma 4. If the transition probability matrix from $h_{k}=s_{1}$ to $h_{k+1}=s$ is $\Pi=\left(\pi_{s_{1}}\right), s, s_{1} \in \phi$, then the transition probability 
matrix from $h_{k-i}=r$ to $h_{k}=s_{1}$ is $\Pi^{(i)}=\left(\pi_{r s_{1}}^{(i)}\right), r, s_{1} \in \phi$, which is a multistep transition probability matrix of the Markov chain. Particularly, when $i=0$, the transition probability matrix is $\Pi^{(0)}=I$.

Proof. The transition probability from $h_{k-i}=r$ to $h_{k}=s_{1}$ is $\pi_{r s_{1}}^{(i)}$, then

$$
\begin{aligned}
\pi_{r s_{1}}^{(i)}= & P\left\{h_{k}=s_{1} \mid h_{k-i}=r\right\} \\
= & P\left\{h_{(k-i)+1+(i-1)}=s_{1} \mid h_{k-i}=r\right\} \\
= & \sum_{l_{1} \in \phi} P\left\{h_{(k-i)+1}=l_{1} \mid h_{k-i}=r\right\} \\
& \times P\left\{h_{(k-i)+1+(i-1)}=s_{1} \mid h_{k-i}=r, h_{(k-i)+1}=l_{1}\right\} \\
= & \sum_{l_{1} \in \phi} P\left\{h_{(k-i)+1}=l_{1} \mid h_{k-i}=r\right\} \\
& \times P\left\{h_{(k-i)+1+(i-1)}=s_{1} \mid h_{(k-i)+1}=l_{1}\right\} \\
= & \sum_{l_{1} \in \phi} \pi_{r l_{1}} \pi_{l_{1} s_{1}}^{(i-1)} \\
= & \sum_{l_{1} \in \phi} \pi_{r l_{1}} \sum_{l_{2} \in \phi} P\left\{h_{(k-i)+2}=l_{2} \mid h_{(k-i)+1}=l_{1}\right\} \\
& \times P\left\{h_{(k-i)+2+(i-2)}=s_{1} \mid h_{(k-i)+2}=l_{2}\right\} \\
= & \sum_{l_{1} \in \phi} \pi_{r l_{1}} \sum_{l_{2} \in \phi} \pi_{l_{1} l_{2}} \pi_{l_{2} s_{1}}^{(i-2)} \\
= & \cdots=\sum_{l_{1} \in \phi l_{2} \in \phi} \ldots \sum_{l_{i-1} \in \phi} \pi_{r l_{1}} \pi_{l_{1} l_{2}} \cdots \pi_{l_{i-1} s_{1}} .
\end{aligned}
$$

From matrix multiplication, the transition probability matrix from $h_{k-i}$ to $h_{k}$ is $\Pi^{(i)}$.

Remark 5. It is noted that (3) to (4) are based on the ineffectiveness theory of Markov chain. With Definition 3, the necessary and sufficient conditions on the stochastic stability of closed-loop system in (7) can be obtained.

Theorem 6. The closed-loop system with completely known transition probabilities in (7) is stochastically stable if and only if there exist the matrices $P(i, r)>0$ such that the following matrix inequalities:

$$
\begin{aligned}
L(i, r)=\sum_{j=0}^{\tau^{s c}} \sum_{s_{2}=1 s_{1}=1}^{3} \sum_{i j}^{3} & \lambda_{i s_{2}}^{(i+1-j)} \pi_{r s_{1}}^{(i)} \\
& \times\left(\bar{A}\left(s_{1}\right)+\bar{B}\left(s_{1}\right) K(i, r) \bar{C}(i)\right)^{T} P\left(j, s_{2}\right) \\
& \times\left(\bar{A}\left(s_{1}\right)+\bar{B}\left(s_{1}\right) K(i, r) \bar{C}(i)\right) \\
& -P(i, r)<0
\end{aligned}
$$

hold for all $i \in \varphi$ and $r \in \phi$.
Proof (sufficiency). For the closed-loop system in (7), consider the Lyapunov function

$$
V(X(k), k)=X^{T}(k) P\left(\tau_{k}^{s c}, h_{k-\tau_{k}^{s c}}\right) X(k) .
$$

Then

$$
\begin{aligned}
\varepsilon\{\Delta V(X(k), k)\} \\
=\varepsilon\{V(X(k+1), k+1) \\
\left.\quad-V(X(k), k) \mid X(k), \tau_{k}^{s c}=i, h_{k-\tau_{k}^{s c}}=r\right\} \\
=\varepsilon\left\{X^{T}(k+1) P\left(\tau_{k+1}^{s c}, h_{k+1-\tau_{k+1}^{s c}}\right)\right. \\
\left.\quad \times X(k+1) \mid X(k), \tau_{k}^{s c}=i, h_{k-\tau_{k}^{s c}}=r\right\} \\
-X^{T}(k) P(i, r) X(k) .
\end{aligned}
$$

Define $\tau_{k+1}^{s c}=j, h_{k}=s_{1}$, and $h_{k+1-\tau_{k+1}^{s c}}=s_{2}$. To evaluate the first term in (13), we need to apply the three probability transition matrices:

$$
\begin{aligned}
& \tau_{k}^{s c} \longrightarrow \tau_{k+1}^{s c}: \Lambda, \\
& h_{k-\tau_{k}^{s c}} \longrightarrow h_{k}: \Pi^{(i)}, \\
& h_{k-\tau_{k}^{s c}} \longrightarrow h_{k+1-\tau_{k+1}^{s c}: \Pi^{(i+1-j)} .}
\end{aligned}
$$

Then, (13) can be written as

$$
\begin{aligned}
& \varepsilon\left\{\Delta V(X(k), k) \mid X(k), \tau_{k}^{s c}=i, h_{k-\tau_{k}^{s c}}=r\right\} \\
&=X^{T}(k)\left(\sum_{j=0 s_{2}=1 \sum_{s_{1}=1}^{s c}}^{3} \sum_{i j}^{3} \pi_{r s_{2}}^{(i+1-j)} \pi_{r s_{1}}^{(i)}\right. \\
& \times\left(\bar{A}\left(s_{1}\right)+\bar{B}\left(s_{1}\right) K(i, r) \bar{C}(i)\right)^{T} \\
& \times P\left(j, s_{2}\right) \\
& \times\left(\bar{A}\left(s_{1}\right)+\bar{B}\left(s_{1}\right) K(i, r) \bar{C}(i)\right) \\
&-P(i, r)) X(k) \\
&=X^{T}(k) L(i, r) X(k)<0 .
\end{aligned}
$$

Thus, if $L(i, r)<0$, then

$$
\begin{aligned}
\varepsilon\{ & \left.\Delta V(X(k), k) \mid X(k), \tau_{k}^{s c}=i, h_{k-\tau_{k}^{s c}}=r\right\} \\
= & \varepsilon\left\{V(X(k+1), k+1) \mid X(k), \tau_{k}^{s c}=i, h_{k-\tau_{k}^{s c}}=r\right\} \\
& -V(X(k), k) \\
\leq & -\lambda_{\min }(-L(i, r)) X(k)^{T} X(k) \\
\leq & -\beta X^{T}(k) X(k) \\
= & -\beta\|X(k)\|^{2},
\end{aligned}
$$


where $\beta=\inf \left\{\lambda_{\min }(-L(i, r)), i \in \varphi, r \in \phi\right\}>0$. From inequality (16), we can see that for any $T \geq 1$,

$$
\begin{aligned}
\varepsilon\{V(X(T+1), T+1)\}-\varepsilon\left\{V\left(X_{0}, 0\right)\right\} \\
\quad \leq-\beta \varepsilon\left\{\sum_{t=0}^{T}\|X(t)\|^{2} \mid X_{0}, \tau_{0}^{s c}, h_{-\tau_{0}^{s c}}\right\} .
\end{aligned}
$$

Furthermore

$$
\begin{aligned}
& \varepsilon\left\{\sum_{t=0}^{T}\|X(t)\|^{2} \mid X_{0}, \tau_{0}^{s c}, h_{-\tau_{0}^{s c}}\right\} \\
& \quad \leq \frac{1}{\beta}\left(\varepsilon\left\{V\left(X_{0}, 0\right)\right\}-\varepsilon\{V(X(T+1), T+1)\}\right) \\
& \quad \leq \frac{1}{\beta} \varepsilon\left\{V\left(X_{0}, 0\right)\right\} .
\end{aligned}
$$

Let $T \rightarrow \infty$, then

$$
\begin{gathered}
\varepsilon\left\{\sum_{t=0}^{\infty}\|X(t)\|^{2} \mid X_{0}, \tau_{0}^{s c}, h_{-\tau_{0}^{s c}}\right\} \leq \frac{1}{\beta} \varepsilon\left\{V\left(X_{0}, 0\right)\right\} \\
=\frac{1}{\beta} X^{T}(0) P\left(\tau_{0}^{s c}, h_{-\tau_{0}^{s c}}\right) X(0) .
\end{gathered}
$$

From Definition 3, the closed-loop system in (7) is stochastically stable.

Necessity. Assume that the closed-loop system in (7) is stochastically stable. Then, we have

$$
\varepsilon\left\{\sum_{k=0}^{\infty}\|X(k)\|^{2} \mid X_{0}, \tau_{0}^{s c}, h_{-\tau_{0}^{s c}}\right\}<X_{0}^{T} W X_{0} .
$$

Define

$$
\begin{aligned}
& X^{T}(t) \widetilde{P}\left(T-t, \tau_{t}^{s c}, h_{t-\tau_{t}^{s c}}\right) X(t) \\
& \quad \triangleq \varepsilon\left\{\sum_{k=t}^{T} X^{T}(k) Q\left(\tau_{k}^{s c}, h_{k-\tau_{k}^{s c}}\right) X(k) \mid X_{t}, \tau_{t}^{s c}, h_{t-\tau_{t}^{s c}}\right\},
\end{aligned}
$$

with $Q\left(\tau_{k}^{s c}, h_{k-\tau_{k}^{s c}}\right)>0$. Assume that $X(k) \neq 0$, since $Q\left(\tau_{k}^{s c}\right.$, $\left.h_{k-\tau_{k}^{s c}}\right)>0$, as $T$ increases, $X^{T}(t) \widetilde{P}\left(T-t, \tau_{t}^{s c}, h_{t-\tau_{t}^{s c}}\right) X(t)$ is monotonically increasing, for $k \geq t$. From $(20), X^{T}(t) \widetilde{P}(T-$ $\left.t, \tau_{t}^{s c}, h_{t-\tau_{t}^{s c}}\right) X(t)$ is upper bounded. Furthermore, its limit exists and can be represented as

$$
\begin{aligned}
& X^{T}(t) P(i, r) X(t) \\
& \triangleq \lim _{T \rightarrow \infty} X^{T}(t) \widetilde{P}\left(T-t, \tau_{t}^{s c}=i, h_{t-\tau_{t}^{s c}}=r\right) X(t) \\
& =\lim _{T \rightarrow \infty} \varepsilon\left\{\sum_{k=t}^{T} X^{T}(k) Q\left(\tau_{k}^{s c}, h_{k-\tau_{k}^{s c}}\right)\right. \\
& \left.\quad \times X(k) \mid X_{t}, \tau_{t}^{s c}=i, h_{t-\tau_{t}^{s c}}=r\right\} .
\end{aligned}
$$

Since this is valid for any $X(t)$, we have

$$
P(i, r)=\lim _{T \rightarrow \infty} \widetilde{P}\left(T-t, \tau_{t}^{s c}=i, h_{t-\tau_{t}^{s c}}=r\right) .
$$

From (22), we have $P(i, r)>0$, since $Q\left(\tau_{k}^{s c}, h_{k-\tau_{k}^{s c}}\right)>0$. Consider

$$
\begin{gathered}
\varepsilon\left\{X^{T}(t) \widetilde{P}\left(T-t, \tau_{t}^{s c}, h_{t-\tau_{t}^{s c}}\right) X(t)-X^{T}(t+1)\right. \\
\times \widetilde{P}\left(T-t-1, \tau_{t+1}^{s c}, h_{t+1-\tau_{t+1}^{s c}}\right) \\
\left.\times X(t+1) \mid X_{t}, \tau_{t}^{s c}=i, h_{t-\tau_{t}^{s c}}=r\right\} \\
=X^{T}(k) Q(i, r) X(k) .
\end{gathered}
$$

By using Lemma 4, the second term in (24) can be written as

$$
\begin{aligned}
\varepsilon\left\{X^{T}(t+1) \widetilde{P}(\right. & \left.T-t-1, \tau_{t+1}^{s c}, h_{t+1-\tau_{t+1}^{s c}}\right) X(t+1) \mid X_{t}, \\
\tau_{t}^{s c}=i, h_{t-\tau_{t}^{s c}} & r\} \\
=X^{T}(t)\{ & \sum_{j=0}^{\tau^{s c}} \sum_{s_{2}=1}^{3} \sum_{s_{1}=1}^{3} \lambda_{i j} \pi_{r s_{2}}^{(i+1-j)} \pi_{r s_{1}}^{(i)} \\
& \times\left(\bar{A}\left(s_{1}\right)+\bar{B}\left(s_{1}\right) K(i, r) \bar{C}(i)\right)^{T} \\
& \times \widetilde{P}\left(T-t-1, j, s_{2}\right) \\
& \left.\times\left(\bar{A}\left(s_{1}\right)+\bar{B}\left(s_{1}\right) K(i, r) \bar{C}(i)\right)\right\} X(t) .
\end{aligned}
$$

Substituting (25) into (24), we can get

$$
\begin{aligned}
& X^{T}(t)\left\{\widetilde{P}\left(T-t, \tau_{t}^{s c}, h_{t-\tau_{t}^{s c}}\right)\right. \\
&-\sum_{j=0}^{\tau} \sum_{s_{2}=1}^{3} \sum_{s_{1}=1}^{3} \lambda_{i j} \pi_{r s_{2}}^{(i+1-j)} \pi_{r s_{1}}^{(i)} \\
& \times\left(\bar{A}\left(s_{1}\right)+\bar{B}\left(s_{1}\right) K(i, r) \bar{C}(i)\right)^{T} \\
& \times P\left(T-t-1, j, s_{2}\right) \\
&\left.\times\left(\bar{A}\left(s_{1}\right)+\bar{B}\left(s_{1}\right) K(i, r) \bar{C}(i)\right)\right\} X(t)
\end{aligned}
$$$$
=X^{T}(t) Q(i, r) X(t) .
$$ 
Letting $T \rightarrow \infty$ and noticing $(22)$ and $Q(i, r)>0$, we have

$$
\begin{aligned}
P(i, r)-\sum_{j=0}^{\tau} \sum_{s_{2}=1}^{3} \sum_{s_{1}=1}^{3} \lambda_{i j} \pi_{r s_{2}}^{(i+1-j)} \pi_{r s_{1}}^{(i)} & \\
& \times\left(\bar{A}\left(s_{1}\right)+\bar{B}\left(s_{1}\right) K(i, r) \bar{C}(i)\right)^{T} \\
& \times P\left(j, s_{2}\right)\left(\bar{A}\left(s_{1}\right)\right. \\
& \left.+\bar{B}\left(s_{1}\right) K(i, r) \bar{C}(i)\right)>0
\end{aligned}
$$

This completes the proof.

Remark 7. Although the proof of Theorem 6 is similar to the literature [10], the considered problem is not the same. The $S-C$ random delays and random sampling periods are considered in this paper; however, the $S-C$ and $C-A$ random delays are considered in [10]. In addition, the continuous systems are considered in this paper and the discrete systems are considered in [10].

Theorem 6 gives the sufficient and necessary conditions on the existence of the state feedback controller, when transition probabilities are completely accessible. However, the conditions in (11) are nonlinear in the controller matrices. In the following theorem, the equivalent LMI conditions to (11) are given.

Theorem 8. There exists a controller (4) such that the closedloop system in (7) is stochastically stable if and only if there exist matrices $K(i, r)$ and $M\left(j, s_{2}\right)>0, P(i, r)>0$ such that the following matrix inequalities

$$
\begin{gathered}
{\left[\begin{array}{cc}
-P(i, r) & V^{T}(i, r) \\
* & -Y(i, r)
\end{array}\right]<0} \\
P\left(j, s_{2}\right) M\left(j, s_{2}\right)=I
\end{gathered}
$$

with

$$
\begin{aligned}
& V(i, r)=\left[\begin{array}{lllll}
V_{0}^{T}(i, r) & V_{1}^{T}(i, r) & \cdots & V_{\tau^{s c}}^{T}(i, r)
\end{array}\right]^{T}, \\
& V_{j}(i, r)=\left[\begin{array}{lll}
V_{j 1}^{T}(i, r) & V_{j 2}^{T}(i, r) & V_{j 3}^{T}(i, r)
\end{array}\right]^{T}, \\
& V_{j s_{2}}(i, r) \\
& =\left[\begin{array}{l}
\left(\lambda_{i j} \pi_{r s_{2}}^{(i+1)} \pi_{r 1}^{(i)}\right)^{1 / 2}[\bar{A}(1)+\bar{B}(1) K(i, r) \bar{C}(i)] \\
\left(\lambda_{i j} \pi_{r s_{2}}^{(i+1)} \pi_{r 2}^{(i)}\right)^{1 / 2}[\bar{A}(2)+\bar{B}(2) K(i, r) \bar{C}(i)] \\
\left(\lambda_{i j} \pi_{r s_{2}}^{(i+1-j)} \pi_{r 3}^{(i)}\right)^{1 / 2}[\bar{A}(3)+\bar{B}(3) K(i, r) \bar{C}(i)]
\end{array}\right], \\
& Y(i, r)=\operatorname{diag}\left\{\begin{array}{llll}
Y_{0}(i, r) & Y_{1}(i, r) & \cdots & Y_{\tau^{s c}}(i, r)
\end{array}\right\}, \\
& Y_{j}(i, r)=\operatorname{diag}\left\{Y_{j 1}(i, r) Y_{j 2}(i, r) \quad Y_{j 3}(i, r)\right\}, \\
& Y_{j s_{2}}(i, r)=\operatorname{diag}\left\{M\left(j, s_{2}\right) M\left(j, s_{2}\right) \quad M\left(j, s_{2}\right)\right\}
\end{aligned}
$$

hold for all $i, j \in \varphi$ and $r, s_{1}$, and $s_{2} \in \phi$.
Proof. By applying the Schur complement and letting $M\left(j, s_{2}\right)=P^{-1}\left(j, s_{2}\right)$, the proof can be readily completed.

Remark 9. The conditions in Theorem 8 are a set of LMIs with nonconvex constraints. This can be solved by several existing iterative LMI algorithms. Using a cone complementarity approach [22-24], we can solve this nonconvex feasibility problem by formulating it into an optimisation problem subject to LMI constraints.

Now, using a cone complementarity approach, we suggest the following nonlinear minimisation problem involving LMI conditions instead of the original nonconvex feasibility problem formulated in Theorem 8 .

Minimise

$$
\operatorname{Tr}\left(\sum_{j=0}^{\tau^{s c}} \sum_{s_{2}=1}^{3} P_{j s_{2}} M_{j s_{2}}\right)
$$

subject to (28) and

$$
\left[\begin{array}{cc}
P\left(j, s_{2}\right) & I \\
I & M\left(j, s_{2}\right)
\end{array}\right] \geq 0
$$

Algorithm 10. (1) Find a feasible point $\left(P_{j s_{2}}(0), M_{j s_{2}}(0)\right)$ satisfying (30). If there are none, set $k=0$.

(2) Solve the following LMI problem

$\operatorname{Min} \operatorname{Tr}\left(\sum_{j=0}^{\tau^{s c}} \sum_{s_{2}=1}^{3}\left(P_{j s_{2}}^{k} M_{j s_{2}}+P_{j s_{2}} M_{j s_{2}}^{k}\right)\right)$ subject to (31).

Set $P_{j s_{2}}^{k+1}=P_{j s_{2}}, M_{j s_{2}}^{k+1}=M_{j s_{2}}$.

(3) If the conditions in (31) are satisfied, then return to (1). If the conditions in (31) are not satisfied within a specified number $\left(k_{\max }\right)$ of iterations, then exit. Otherwise, set $k=k+1$ and go to (2).

\section{The Case of Partly Known Transition Probabilities}

In the above section, the transition probabilities are assumed completely accessible and considered as the available knowledge for analyzing the NCSs. In practice, this kind of information including the variation of time delay and actual sampling period is hard to obtain. This variation can degrade the control performance and even make the system unstable.

In this section, the transition probabilities are assumed partially accessible. The transition probabilities in (3) are considered to be partially available; namely, some elements in matrices $\Pi$ and $\Lambda$ are time invariant but unknown. For instance,

$$
\Pi=\left[\begin{array}{ccc}
\pi_{11} & ? & ? \\
? & \pi_{22} & ? \\
\pi_{31} & \pi_{32} & \pi_{33}
\end{array}\right], \quad \Lambda=\left[\begin{array}{cccc}
\lambda_{11} & ? & \lambda_{13} & ? \\
? & \lambda_{22} & ? & \lambda_{24} \\
? & ? & \lambda_{33} & \lambda_{34} \\
\lambda_{41} & \lambda_{42} & \lambda_{43} & \lambda_{44}
\end{array}\right]
$$


where ? represents the unavailable elements. For notation clarity, $i \in \varphi, r \in \phi$, we denote

$$
\begin{aligned}
\varphi_{\chi}^{i} & =\left\{j: \text { if } \lambda_{i j} \text { is known }\right\}, \\
\varphi_{\mu \chi}^{i} & =\left\{j: \text { if } \lambda_{i j} \text { is unknown }\right\}, \\
\phi_{\chi}^{r} & =\left\{s: \text { if } \pi_{r s} \text { is known }\right\}, \\
\phi_{\mu \chi}^{r} & =\left\{s: \text { if } \pi_{r s} \text { is unknown }\right\} .
\end{aligned}
$$

Moreover, if $\varphi_{\chi}^{i} \neq 0, \phi_{\chi}^{r} \neq 0$, it is further described as

$$
\begin{aligned}
\varphi_{\chi}^{i} & =\left\{\chi_{0}^{i}, \chi_{1}^{i}, \ldots, \chi_{m}^{i}\right\}, \quad 0 \leq m \leq \tau^{s c} ; \\
\phi_{\chi}^{r} & =\left\{\chi_{1}^{r}, \ldots, \chi_{n}^{r}\right\}, \quad 1 \leq n \leq 3, \\
\varphi_{\mu \chi}^{i} & =\left\{\bar{\chi}_{1}^{i}, \bar{\chi}_{2}^{i}, \ldots, \bar{\chi}_{\tau^{s c}-m}^{i}\right\}, \quad 0 \leq m \leq \tau^{s c}, \\
\phi_{\mu \chi}^{r} & =\left\{\bar{\chi}_{1}^{r}, \ldots, \bar{\chi}_{3-n}^{r}\right\}, \quad 1 \leq n \leq 3,
\end{aligned}
$$

where $\chi_{m}^{i}$ represents the $m$ th known element with the index in the $i$ th row of matrix $\Lambda, \bar{\chi}_{j}^{i}\left(j=1,2, \ldots, \tau^{s c}-m\right)$ represents the $j$ th unknown element with the index in the $i$ th row of matrix $\Lambda, \chi_{n}^{r}$ represents the $n$th known element with the index in the $r$ th row of matrix $\Pi$, and $\bar{\chi}_{j}^{r}(j=1,2, \ldots, 3-n)$ represents the $j$ th unknown element with the index in the $r$ th row of matrix $\Pi$.

Remark 11. Note that the expression of (33)-(35) was first introduced for regular state-space Markovian systems $[13,14]$, and it covers completely known and completely unknown probabilities. If $\varphi_{\chi}^{i}=\varphi, \varphi_{\mu \chi}^{i}=0$ or $\phi_{\chi}^{r}=\phi$, and $\phi_{\mu \chi}^{r}=0$, the transition probabilities are completely available. If $\varphi_{\chi}^{i}=$ $0, \varphi_{\mu \chi}^{i}=\varphi$ or $\phi_{\chi}^{r}=0$, and $\phi_{\mu \chi}^{r}=\phi$, the transition probabilities are completely unavailable.

Now, the following theorem presents a sufficient condition for the stochastic stability of the system described by (7) with partially known transition probabilities (33).

Theorem 12. The system described by (7) with partially known transition probabilities (33) is stochastically stable if there exist matrices $P(i, r)>0$ such that the following matrix inequalities

$$
\begin{gathered}
\left(\sum_{j \in \varphi_{\chi}^{i} s_{1} \in \phi_{X}^{r}}\left(\sum_{s_{2} \in \phi_{X}^{r}} \lambda_{i j} \pi_{r s_{2}}^{(i+1-j)} \pi_{r s_{1}}^{(i)}+\sum_{s_{2} \in \phi_{\mu \chi}^{r}} \lambda_{i j} \pi_{r}^{(i+1-j)} \pi_{r s_{1}}^{(i)}\right)\right. \\
+\sum_{j \in \varphi_{\chi}^{i} s_{1} \in \phi_{\mu \chi}^{r}}\left(\sum_{s_{2} \in \phi_{\chi}^{r}} \lambda_{i j} \pi_{r s_{2}}^{(i+1-j)} \pi_{r}^{(i)}+\sum_{s_{2} \in \phi_{\mu \chi}^{r}} \lambda_{i j} \pi_{r}^{(i+1-j)} \pi_{r}^{(i)}\right)
\end{gathered}
$$

$$
\begin{aligned}
& +\sum_{j \in \varphi_{\mu \chi}^{i}} \sum_{s_{1} \in \phi_{X}^{r}}\left(\sum_{s_{2} \in \phi_{\chi}^{r}} \lambda_{i} \pi_{r s_{2}}^{(i+1-j)} \pi_{r s_{1}}^{(i)}+\sum_{s_{2} \in \phi_{\mu \chi}^{r}} \lambda_{i} \pi_{r}^{(i+1-j)} \pi_{r s_{1}}^{(i)}\right) \\
& \left.\left.+\sum_{j \in \varphi_{\mu \chi}^{i} s_{1} \in \phi_{\mu \chi}^{r}} \sum_{s_{2} \in \phi_{\chi}^{r}} \lambda_{i} \pi_{r s_{2}}^{(i+1-j)} \pi_{r}^{(i)}+\sum_{s_{2} \in \phi_{\mu \chi}^{r}} \lambda_{i} \pi_{r}^{(i+1-j)} \pi_{r}^{(i)}\right)\right) \\
& * \widetilde{A}^{T}\left(i, r, s_{1}\right) P\left(j, s_{2}\right) \widetilde{A}\left(i, r, s_{1}\right)-P(i, r)<0, \quad i=0,1,2,
\end{aligned}
$$

$$
\begin{aligned}
& \left(\sum_{j \in \varphi_{\chi}^{i-2}} \sum_{s_{1}=1}^{3}\left(\sum_{s_{2} \in \phi_{\chi}^{r}} \lambda_{i j}+\sum_{s_{2} \in \phi_{\mu \chi}^{r}} \lambda_{i j}\right)\right. \\
& +\sum_{j \in \varphi_{\mu \chi}^{i-2} s_{1}=1}^{3}\left(\sum_{s_{2} \in \phi_{\chi}^{r}} \lambda_{i}+\sum_{s_{2} \in \phi_{\mu \chi}^{r}} \lambda_{i}\right) \\
& +\sum_{j \in \bar{\varphi}_{\chi}^{i-2} \sum_{s_{1}=1}}^{3}\left(\sum_{s_{2} \in \phi_{\chi}^{r}} \lambda_{i j} \pi_{r s_{2}}^{(i+1-j)}+\sum_{s_{2} \in \phi_{\mu \chi}^{r}} \lambda_{i j} \pi_{r}^{(i+1-j)}\right) \\
& \left.+\sum_{j \in \bar{\varphi}_{\mu \chi}^{i-2} s_{1}=1} \sum_{s_{2} \in \phi_{\chi}^{r}}^{3}\left(\sum_{i s_{2}} \pi_{s_{2} \in \phi_{\mu \chi}^{r}}^{(i+1-j)}+\sum_{i} \pi_{r}^{(i+1-j)}\right)\right) \\
& * \widetilde{A}^{T}\left(i, r, s_{1}\right) P\left(j, s_{2}\right) \widetilde{A}\left(i, r, s_{1}\right)-P(i, r)<0, \quad 3 \leq i \leq \tau^{s c}
\end{aligned}
$$

hold for all $i \in \varphi$ and $r \in \phi$, where

$$
\begin{aligned}
\widetilde{A}\left(i, r, s_{1}\right) & =\bar{A}\left(s_{1}\right)+\bar{B}\left(s_{1}\right) K(i, r) \bar{C}(i), \\
\lambda_{i} & =1-\sum_{j \in \varphi_{\chi}^{i}} \lambda_{i j}, \quad \pi_{r}^{(i+1-j)}=1-\sum_{s_{2} \in \phi_{\chi}^{r}} \pi_{r s_{2}}^{(i+1-j)}, \\
\pi_{r}^{(i)} & =1-\sum_{s_{1} \in \phi_{\chi}^{r}} \pi_{r s_{1}}^{(i)}, \\
\varphi_{\chi}^{i-2} & =\left\{j: \text { if } \lambda_{i j} \text { is known and } j \leq i-2\right\}, \\
\varphi_{\mu \chi}^{i-2} & =\left\{j: \text { if } \lambda_{i j} \text { is unknown and } j \leq i-2\right\}, \\
\bar{\varphi}_{\chi}^{i-2} & =\left\{j: \text { if } \lambda_{i j} \text { is known and } j>i-2\right\}, \\
\bar{\varphi}_{\mu \chi}^{i-2} & =\left\{j: \text { if } \lambda_{i j} \text { is unknown and } j>i-2\right\} .
\end{aligned}
$$

Proof. From Theorem 6, we know

$$
\begin{gathered}
L(i, r)=\sum_{j=0}^{\tau^{s c}} \sum_{s_{2}=1}^{3} \sum_{s_{1}=1}^{3} \lambda_{i j} \pi_{r s_{2}}^{(i+1-j)} \pi_{r s_{1}}^{(i)} \widetilde{A}^{T}\left(i, r, s_{1}\right) P\left(j, s_{2}\right) \\
\times \widetilde{A}\left(i, r, s_{1}\right)-P(i, r)<0 .
\end{gathered}
$$


However, from (30), we know that some elements are inaccessible in $\Lambda$ and $\Pi$. According to the property of Markov chains, we obtain

$$
\begin{aligned}
& \bar{P}(j, r)= \sum_{s_{2}=1}^{3} \pi_{r s_{2}}^{(i+1-j)} P\left(j, s_{2}\right) \\
&= \sum_{s_{2} \in \phi_{x}^{r}} \pi_{r s_{2}}^{(i+1-j)} P\left(j, s_{2}\right)+\sum_{s_{2} \in \phi_{\mu x}^{r}} \pi_{r s_{2}}^{(i+1-j)} P\left(j, s_{2}\right) \\
& \leq \sum_{s_{2} \in \phi_{x}^{r}} \pi_{r s_{2}}^{(i+1-j)} P\left(j, s_{2}\right)+\sum_{s_{2} \in \phi_{\mu x}^{r}} \pi_{r}^{(i+1-j)} P\left(j, s_{2}\right), \\
& \widetilde{P}(i, j, r)= \sum_{s_{1}=1}^{3} \pi_{r s_{1}}^{(i)} \widetilde{A}^{T}\left(i, r, s_{1}\right) \bar{P}(j, r) \widetilde{A}\left(i, r, s_{1}\right) \\
&= \sum_{s_{1} \in \phi_{x}^{r}} \pi_{r s_{1}}^{(i)} \widetilde{A}^{T}\left(i, r, s_{1}\right) \bar{P}(j, r) \widetilde{A}\left(i, r, s_{1}\right) \\
&+\sum_{s_{1} \in \phi_{\mu x}^{r}} \pi_{r s_{1}}^{(i)} \widetilde{A}^{T}\left(i, r, s_{1}\right) \bar{P}(j, r) \widetilde{A}\left(i, r, s_{1}\right) \\
& \leq \sum_{s_{1} \in \phi_{x}^{r}} \pi_{r s_{1}}^{(i)} \widetilde{A}^{T}\left(i, r, s_{1}\right) \bar{P}(j, r) \widetilde{A}\left(i, r, s_{1}\right) \\
&+\sum_{s_{1} \in \phi_{\mu x}^{r}} \pi_{r}^{(i)} \widetilde{A}^{T}\left(i, r, s_{1}\right) \bar{P}(j, r) \widetilde{A}\left(i, r, s_{1}\right) \\
& \leq \sum_{j \in \varphi_{x}^{i}} \lambda_{i j} \widetilde{P}(i, r, j)+\sum_{j \in \varphi_{\mu x}^{i}} \lambda_{i} \widetilde{P}(i, r, j) \\
& \sum_{j=0} \lambda_{i j} \widetilde{P}(i, j, r)= \sum_{j \in \varphi_{x}^{i}} \lambda_{i j} \widetilde{P}(i, r, j)+\sum_{j \in \varphi_{\mu x}^{i}} \lambda_{i j} \widetilde{P}(i, r, j) \\
& \tau^{s c}
\end{aligned}
$$

where $\widetilde{A}\left(i, r, s_{1}\right), \lambda_{i}, \pi_{r}^{(i+1-j)}, \pi_{r}^{(i)}$ are defined in (38).

According to Lemma $4, \pi_{r s}^{(3)}=\sum_{l_{2}} \sum_{l_{1}} \pi_{r l_{1}} \pi_{l_{1} l_{2}} \pi_{l_{2} s}$. We obtain $\pi_{r s}^{(3)}$ (for all $r, s \in \phi$ ), that is inaccessible in $\Pi^{(3)}$, even if there is an inaccessible element in $\Pi$. So we obtain $\pi_{r s}^{(i)}$ (for all $r, s \in \phi, i \geq 3$ ), that is inaccessible in $\Pi^{(i)}$, and

$$
\sum_{s \in \phi_{\mu \chi}^{r}} \pi_{r s}^{(i)}=\sum_{s \in \phi} \pi_{r s}^{(i)}=1
$$

Substituting the formulae (40)-(43) into (39), we can obtain (36) and (37). This completes the proof.

Theorem 12 gives the sufficient conditions on the existence of the state feedback controller, when transition probabilities are partly accessible. However, the conditions in (36) and (37) are nonlinear in the controller matrices. In the following theorem, the equivalent LMI conditions to (36) and (37) are given.
Theorem 13. Consider the system described by (7) with partially known transition probabilities (30), the corresponding system is stochastically stable if there exist matrices $K(i, r)$ and $X\left(\chi_{c}^{i}, \chi_{a}^{r}\right)>0, X\left(\bar{\chi}_{d}^{i}, \bar{\chi}_{b}^{r}\right)>0, X\left(\chi_{c}^{i}, \bar{\chi}_{b}^{r}\right)>0, X\left(\bar{\chi}_{d}^{i}, \chi_{a}^{r}\right)>0$, $X\left(\chi_{g}^{i-2}, \chi_{a}^{r}\right)>0, X\left(\chi_{g}^{i-2}, \bar{\chi}_{b}^{r}\right)>0, X\left(\chi_{e}^{i-2}, \chi_{a}^{r}\right)>0, X\left(\chi_{e}^{i-2}\right.$, $\left.\bar{\chi}_{b}^{r}\right)>0, X\left(\bar{\chi}_{f}^{i-2}, \chi_{a}^{r}\right)>0, X\left(\bar{\chi}_{f}^{i-2}, \bar{\chi}_{b}^{r}\right)>0, X\left(\bar{\chi}_{h}^{i-2}, \chi_{a}^{r}\right)>$ $0, X\left(\bar{\chi}_{h}^{i-2}, \bar{\chi}_{b}^{r}\right)>0, P\left(\chi_{c}^{i}, \chi_{a}^{r}\right)>0, P\left(\bar{\chi}_{d}^{i}, \bar{\chi}_{b}^{r}\right)>0, P\left(\chi_{c}^{i}\right.$, $\left.\bar{\chi}_{b}^{r}\right)>0, P\left(\bar{\chi}_{d}^{i}, \chi_{a}^{r}\right)>0, P\left(\chi_{g}^{i-2}, \chi_{a}^{r}\right)>0, P\left(\chi_{g}^{i-2}, \bar{\chi}_{b}^{r}\right)>$ $0, P\left(\chi_{e}^{i-2}, \chi_{a}^{r}\right)>0, P\left(\chi_{e}^{i-2}, \bar{\chi}_{b}^{r}\right)>0, P\left(\bar{\chi}_{f}^{i-2}, \chi_{a}^{r}\right)>0, P\left(\bar{\chi}_{f}^{i-2}\right.$, $\left.\bar{\chi}_{b}^{r}\right)>0, P\left(\bar{\chi}_{h}^{i-2}, \chi_{a}^{r}\right)>0, P\left(\bar{\chi}_{h}^{i-2}, \bar{\chi}_{b}^{r}\right)>0$, such that the following matrix inequalities:

$$
\begin{aligned}
& {\left[\begin{array}{ccc}
-P(i, r) & V^{T}(i, r) & \widetilde{V}^{T}(i, r) \\
* & -Y(i, r) & 0 \\
* & * & -\widetilde{Y}(i, r)
\end{array}\right]<0,} \\
& P\left(\chi_{c}^{i}, \chi_{a}^{r}\right) X\left(\chi_{c}^{i}, \chi_{a}^{r}\right)=I, \\
& P\left(\chi_{c}^{i}, \chi_{a}^{r}\right) X\left(\chi_{c}^{i}, \bar{\chi}_{b}^{r}\right)=I, \\
& P\left(\bar{\chi}_{d}^{i}, \chi_{a}^{r}\right) X\left(\bar{\chi}_{d}^{i}, \chi_{a}^{r}\right)=I, \\
& P\left(\bar{\chi}_{d}^{i}, \bar{\chi}_{b}^{r}\right) X\left(\bar{\chi}_{d}^{i}, \bar{\chi}_{b}^{r}\right)=I, \\
& P\left(\chi_{g}^{i-2}, \chi_{a}^{r}\right) X\left(\chi_{g}^{i-2}, \chi_{a}^{r}\right)=I, \\
& P\left(\chi_{g}^{i-2}, \bar{\chi}_{b}^{r}\right) X\left(\chi_{g}^{i-2}, \bar{\chi}_{b}^{r}\right)=I, \\
& P\left(\chi_{e}^{i-2}, \chi_{a}^{r}\right) X\left(\chi_{e}^{i-2}, \chi_{a}^{r}\right)=I, \\
& P\left(\chi_{e}^{i-2}, \bar{\chi}_{b}^{r}\right) X\left(\chi_{e}^{i-2}, \bar{\chi}_{b}^{r}\right)=I, \\
& P\left(\bar{\chi}_{f}^{i-2}, \chi_{a}^{r}\right) X\left(\bar{\chi}_{f}^{i-2}, \chi_{a}^{r}\right)=I, \\
& P\left(\bar{\chi}_{f}^{i-2}, \bar{\chi}_{b}^{r}\right) X\left(\bar{\chi}_{f}^{i-2}, \bar{\chi}_{b}^{r}\right)=I, \\
& P\left(\bar{\chi}_{h}^{i-2}, \chi_{a}^{r}\right) X\left(\bar{\chi}_{h}^{i-2}, \chi_{a}^{r}\right)=I, \\
& P\left(\bar{\chi}_{h}^{i-2}, \bar{\chi}_{b}^{r}\right) X\left(\bar{\chi}_{h}^{i-2}, \bar{\chi}_{b}^{r}\right)=I
\end{aligned}
$$

with

$$
\begin{aligned}
i & =0,1,2, \\
V^{T}(i, r) & =\left[\begin{array}{lll}
V_{1}^{T}(i, r) & V_{2}^{T}(i, r)
\end{array}\right], \\
\widetilde{V}^{T}(i, r) & =\left[\begin{array}{llll}
\widetilde{V}_{1}^{T}(i, r) & \widetilde{V}_{2}^{T}(i, r)
\end{array}\right], \\
V_{i}(i, r) & =\left[\begin{array}{llll}
V_{i \chi_{0}^{i}}^{T}(i, r) & V_{i \chi_{1}^{i}}^{T}(i, r) & \cdots & V_{i \chi_{m}^{i}}^{T}(i, r)
\end{array}\right]^{T}, \\
\widetilde{V}_{i}(i, r) & =\left[\begin{array}{llll}
\widetilde{V}_{i \bar{\chi}_{1}^{i}}^{T}(i, r) & \widetilde{V}_{i \bar{\chi}_{2}^{i}}^{T}(i, r) & \cdots & \widetilde{V}_{\bar{\chi}_{\tau}^{i} c_{c-m}}^{T}(i, r)
\end{array}\right]^{T}, \\
V_{1 \chi_{c}^{i}}(i, r) & =\left[\begin{array}{llll}
V_{1 \chi_{c}^{i} \chi_{1}^{r}}^{T}(i, r) & V_{1 \chi_{c}^{i} \chi_{2}^{r}}^{T}(i, r) & \cdots & V_{1 \chi_{c}^{i} \chi_{n}^{r}}^{T}(i, r)
\end{array}\right]^{T}, \\
V_{2 \chi_{a}^{r}}(i, r) & =\left[\begin{array}{llll}
V_{2 \chi_{c}^{i} \bar{\chi}_{1}^{r}}^{T}(i, r) & V_{2 \chi_{c}^{i} \bar{\chi}_{2}^{r}}^{T}(i, r) & \cdots & V_{2 \chi_{c}^{i} \bar{\chi}_{3-n}^{r}}^{T}(i, r)
\end{array}\right]^{T},
\end{aligned}
$$




$$
\begin{aligned}
& \widetilde{V}_{1 \bar{\chi}_{d}^{i}}(i, r)=\left[\begin{array}{lllll}
\widetilde{V}_{1}^{T} \bar{\chi}_{d}^{i} \chi_{1}^{r} & (i, r) & \widetilde{V}_{1 \bar{\chi}_{d}^{i} \chi_{2}^{r}}^{T}(i, r) & \cdots & \widetilde{V}_{1 \bar{\chi}_{d}^{i} \chi_{n}^{r}}^{T}(i, r)
\end{array}\right]^{T}, \quad \widetilde{Y}_{i}(i, r)=\operatorname{diag}\left\{\widetilde{Y}_{i \bar{\chi}_{1}^{i}}(i, r),\right. \\
& \widetilde{V}_{2 \bar{\chi}_{d}^{i}}(i, r)=\left[\begin{array}{lllll}
\widetilde{V}_{2 \bar{\chi}_{d}^{i} \bar{\chi}_{1}^{r}}^{T}(i, r) & \widetilde{V}_{2 \bar{\chi}_{d}^{i} r_{2}^{r}}^{T}(i, r) & \cdots & \widetilde{V}_{2 \bar{\chi}_{d}^{i} \bar{X}_{3-n}^{r}}^{T}(i, r)
\end{array}\right]^{T}, \\
& \left.\widetilde{Y}_{i \bar{\chi}_{2}^{i}}(i, r), \ldots, \widetilde{Y}_{i \bar{\chi}_{\tau}^{i} c_{-m}}(i, r)\right\},
\end{aligned}
$$

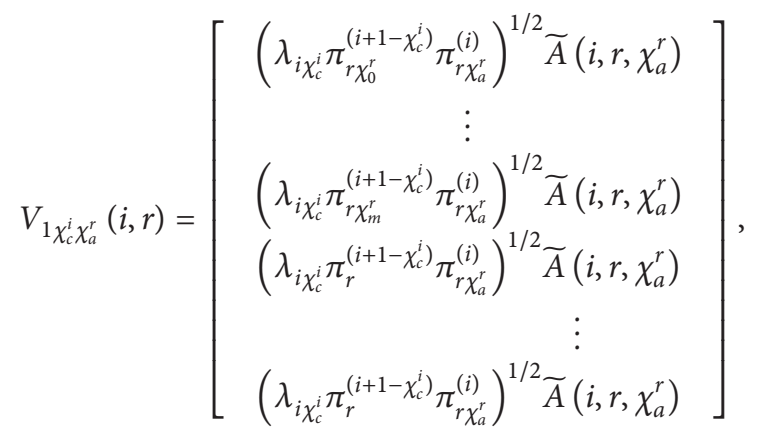

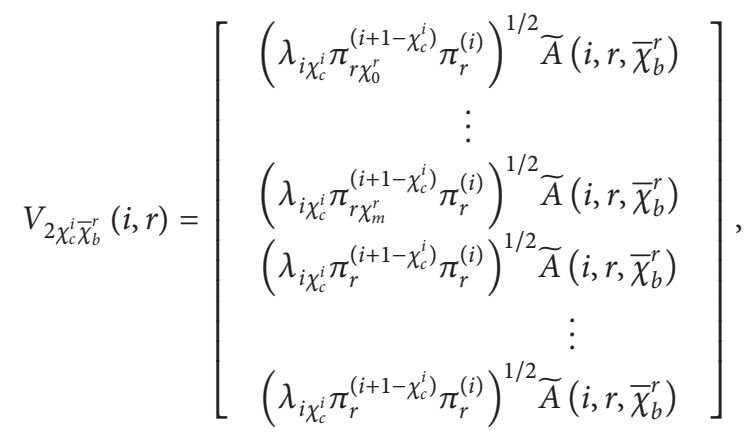

$$
\begin{aligned}
& \widetilde{V}_{1 \bar{\chi}_{d}^{i} \chi_{a}^{r}}(i, r)=\left[\begin{array}{c}
\left(\lambda_{i} \pi_{r \chi_{0}^{r}}^{\left(i+1-\bar{\chi}_{d}^{i}\right)} \pi_{r \chi_{a}^{r}}^{(i)}\right)^{1 / 2} \widetilde{A}\left(i, r, \chi_{a}^{r}\right) \\
\vdots \\
\left(\lambda_{i} \pi_{r \chi_{m}^{r}}^{\left(i+1-\bar{\chi}_{d}^{i}\right)} \pi_{r \chi_{a}^{r}}^{(i)}\right)^{1 / 2} \widetilde{A}\left(i, r, \chi_{a}^{r}\right) \\
\left(\lambda_{i} \pi_{r}^{\left(i+1-\bar{\chi}_{d}^{i}\right)} \pi_{r \chi_{a}^{r}}^{(i)}\right)^{1 / 2} \widetilde{A}\left(i, r, \chi_{a}^{r}\right) \\
\vdots \\
\left(\lambda_{i} \pi_{r}^{\left(i+1-\bar{\chi}_{d}^{i}\right)} \pi_{r \chi_{a}^{r}}^{(i)}\right)^{1 / 2} \widetilde{A}\left(i, r, \chi_{a}^{r}\right)
\end{array}\right], \\
& \widetilde{V}_{2 \bar{\chi}_{d}^{i} \bar{\chi}_{b}^{r}}(i, r)=\left[\begin{array}{c}
\left(\lambda_{i} \pi_{r \chi_{0}^{r}}^{\left(i+1-\bar{\chi}_{d}^{i}\right)} \pi_{r}^{(i)}\right)^{1 / 2} \widetilde{A}\left(i, r, \bar{\chi}_{b}^{r}\right) \\
\vdots \\
\left(\lambda_{i} \pi_{r \chi_{m}^{r}}^{\left(i+1-\bar{\chi}_{d}^{i}\right)} \pi_{r}^{(i)}\right)^{1 / 2} \widetilde{A}\left(i, r, \bar{\chi}_{b}^{r}\right) \\
\left(\lambda_{i} \pi_{r}^{\left(i+1-\bar{\chi}_{d}^{i}\right)} \pi_{r}^{(i)}\right)^{1 / 2} \widetilde{A}\left(i, r, \bar{\chi}_{b}^{r}\right) \\
\vdots \\
\left(\lambda_{i} \pi_{r}^{\left(i+1-\bar{\chi}_{d}^{i}\right)} \pi_{r}^{(i)}\right)^{1 / 2} \widetilde{A}\left(i, r, \bar{\chi}_{b}^{r}\right)
\end{array}\right], \\
& Y(i, r)=\operatorname{diag}\left\{Y_{1}(i, r), Y_{2}(i, r)\right\}, \\
& \widetilde{Y}(i, r)=\operatorname{diag}\left\{\widetilde{Y}_{1}(i, r), \widetilde{Y}_{2}(i, r)\right\}, \\
& Y_{i}(i, r)=\operatorname{diag}\left\{Y_{i \chi_{0}^{i}}(i, r)\right. \text {, } \\
& \left.Y_{i \chi_{1}^{i}}(i, r), \ldots, Y_{i \chi_{m}^{i}}(i, r)\right\} \\
& Y_{1 \chi_{c}^{i}}(i, r)=\operatorname{diag}\left\{Y_{1 \chi_{c}^{i} \chi_{1}^{r}}(i, r),\right. \\
& \left.Y_{1 \chi_{c}^{i} \chi_{2}^{r}}(i, r), \ldots, Y_{1 \chi_{c}^{i} \chi_{n}^{r}}(i, r)\right\}, \\
& Y_{2 \chi_{a}^{r}}(i, r)=\operatorname{diag}\left\{Y_{2 \chi_{c}^{i} \bar{\chi}_{1}^{r}}(i, r),\right. \\
& \left.Y_{2 \chi_{c}^{i} \bar{\chi}_{2}^{r}}(i, r), \ldots, Y_{2 \chi_{c}^{i} \bar{X}_{3-n}^{r}}(i, r)\right\}, \\
& Y_{1 \chi_{c}^{i} \chi_{a}^{r}}(i, r)=Y_{2 \chi_{a}^{r} \bar{\chi}_{b}^{r}}(i, r) \\
& =\operatorname{diag}\left\{X\left(\chi_{c}^{i}, \chi_{0}^{r}\right), \ldots, X\left(\chi_{c}^{i}, \chi_{m}^{r}\right),\right. \\
& \left.X\left(\chi_{c}^{i}, \bar{\chi}_{1}^{r}\right), \ldots, X\left(\chi_{c}^{i}, \bar{\chi}_{\tau^{s c}-m}^{r}\right)\right\}, \\
& \widetilde{Y}_{1 \bar{\chi}_{d}^{i}}(i, r)=\operatorname{diag}\left\{\widetilde{Y}_{1 \bar{\chi}_{d}^{i} \chi_{1}^{r}}(i, r),\right. \\
& \left.\widetilde{Y}_{1 \bar{\chi}_{d}^{i} \chi_{2}^{r}}(i, r), \ldots, \widetilde{Y}_{1 \bar{\chi}_{d}^{i} \chi_{n}^{r}}(i, r)\right\}, \\
& \widetilde{Y}_{2 \bar{\chi}_{b}^{r}}(i, r)=\operatorname{diag}\left\{\widetilde{Y}_{2 \bar{\chi}_{d}^{i} \bar{\chi}_{1}^{r}}(i, r),\right. \\
& \left.\widetilde{Y}_{2 \bar{\chi}_{d}^{i} \bar{X}_{2}^{r}}(i, r), \ldots, \widetilde{Y}_{2 \bar{\chi}_{d}^{i} \bar{\chi}_{3-n}^{r}}(i, r)\right\}, \\
& \widetilde{Y}_{1 \bar{\chi}_{d}^{i} \chi_{a}^{r}}(i, r)=\widetilde{Y}_{2 \bar{\chi}_{b}^{r} \bar{\chi}_{b}^{r}}(i, r) \\
& =\operatorname{diag}\left\{X\left(\bar{\chi}_{d}^{i}, \chi_{0}^{r}\right), \ldots, X\left(\bar{\chi}_{d}^{i}, \chi_{m}^{r}\right)\right. \text {, } \\
& \left.X\left(\bar{\chi}_{d}^{i}, \bar{\chi}_{1}^{r}\right), \ldots, X\left(\bar{\chi}_{d}^{i}, \bar{\chi}_{\tau^{s c}-m}^{r}\right)\right\}, \\
& 3 \leq i \leq \tau^{s c} \\
& V^{T}(i, r)=\left[\begin{array}{lll}
V_{1}^{T}(i, r) & V_{2}^{T}(i, r)
\end{array}\right], \\
& \widetilde{V}^{T}(i, r)=\left[\begin{array}{lll}
\widetilde{V}_{1}^{T}(i, r) & \widetilde{V}_{2}^{T}(i, r)
\end{array},\right. \\
& V_{1}(i, r)=\left[\begin{array}{llll}
V_{1 \chi_{0}^{i-2}}^{T}(i, r) & \cdots & V_{1 \chi_{\bar{m}}^{i-2}}^{T}(i, r)
\end{array}\right]^{T}, \\
& V_{2}(i, r)=\left[\begin{array}{llll}
V_{2 \chi_{\bar{m}+1}^{i-2}}^{T}(i, r) & \cdots & V_{2 \chi_{m}^{i-2}}^{T}(i, r)
\end{array}\right]^{T}, \\
& \widetilde{V}_{1}(i, r)=\left[\begin{array}{lll}
\widetilde{V}_{1 \bar{\chi}_{1}^{i-2}}^{T}(i, r) & \cdots & \widetilde{V}_{1 \bar{\chi}_{\bar{m}}^{i-2}}^{T}(i, r)
\end{array}\right]^{T},
\end{aligned}
$$

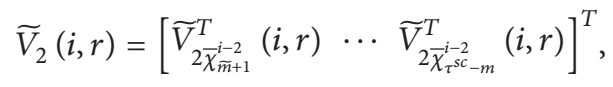

$$
\begin{aligned}
& V_{1 \chi_{g}^{i-2}}(i, r) \\
& =\left[\begin{array}{lll}
V_{1 \chi_{g}^{i-2} 1}^{T}(i, r) & V_{1 \chi_{g}^{i-2} 2}^{T}(i, r) & V_{1 \chi_{g}^{i-2} 3}^{T}(i, r)
\end{array}\right]^{T}, \\
& V_{2 \bar{\chi}_{h}^{i-2}}(i, r) \\
& =\left[\begin{array}{lll}
V_{2 \bar{\chi}_{h}^{i-2} 1}^{T}(i, r) & V_{2 \bar{\chi}_{h}^{i-2} 2}^{T}(i, r) & V_{2 \bar{\chi}_{h}^{i-2} 3}^{T}(i, r)
\end{array}\right]^{T},
\end{aligned}
$$




$$
\begin{aligned}
& \widetilde{V}_{1 \chi_{e}^{i-2}}(i, r) \\
& =\left[\begin{array}{llll}
\widetilde{V}_{1 \chi_{e}^{i-2} 1}^{T}(i, r) & \widetilde{V}_{1 \chi_{e}^{i-2} 2}^{T}(i, r) & \widetilde{V}_{1 \chi_{e}^{i-2} 3}^{T}(i, r)
\end{array}\right]^{T}, \\
& \widetilde{V}_{2 \bar{\chi}_{f}^{i-2}}(i, r) \\
& =\left[\begin{array}{llll}
\widetilde{V}_{2 \bar{\chi}_{f}^{i-2} 1}^{T}(i, r) & \widetilde{V}_{2 \bar{\chi}_{f}^{i-2} 2}^{T}(i, r) & \widetilde{V}_{2 \bar{\chi}_{f}^{i-2} 3}^{T}(i, r)
\end{array}\right]^{T}, \\
& V_{1 \chi_{g}^{i-2} s_{1}}(i, r)=\left[\begin{array}{c}
\left(\lambda_{i \chi_{g}^{i-2}}\right)^{1 / 2} \widetilde{A}\left(i, r, s_{1}\right) \\
\vdots \\
\left(\lambda_{\left.i \chi_{g}^{i-2}\right)^{1 / 2} \widetilde{A}\left(i, r, s_{1}\right)}\right. \\
\left(\lambda_{i \chi_{g}^{i-2}}\right)^{1 / 2} \widetilde{A}\left(i, r, s_{1}\right) \\
\vdots \\
\left(\lambda_{i \chi_{g}^{i-2}}\right)^{1 / 2} \widetilde{A}\left(i, r, s_{1}\right)
\end{array}\right], \\
& V_{2 \bar{\chi}_{h}^{i-2} s_{1}}(i, r)=\left[\begin{array}{c}
\left(\lambda_{i}\right)^{1 / 2} \widetilde{A}\left(i, r, s_{1}\right) \\
\vdots \\
\left(\lambda_{i}\right)^{1 / 2} \widetilde{A}\left(i, r, s_{1}\right) \\
\left(\lambda_{i}\right)^{1 / 2} \widetilde{A}\left(i, r, s_{1}\right) \\
\vdots \\
\left(\lambda_{i}\right)^{1 / 2} \tilde{A}\left(i, r, s_{1}\right)
\end{array}\right] \text {, }
\end{aligned}
$$

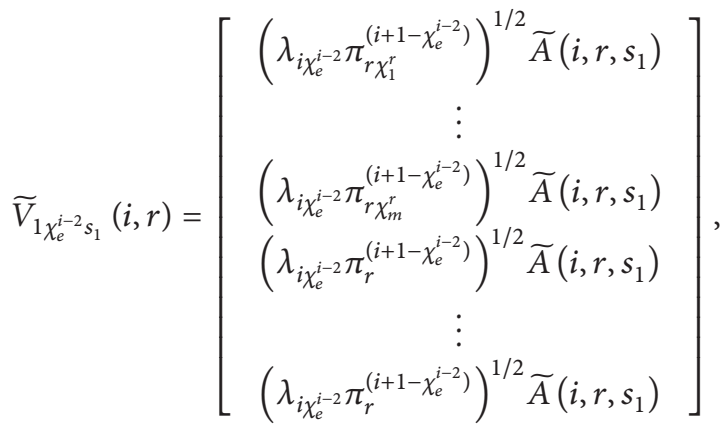

$$
\begin{aligned}
& \widetilde{V}_{2 \bar{\chi}_{f}^{i-2} s_{1}}(i, r)=\left[\begin{array}{c}
\left(\lambda_{i} \pi_{r \chi_{0}^{s c}}^{\left(i+1-\bar{\chi}_{f}^{i-2}\right)}\right)^{1 / 2} \widetilde{A}\left(i, r, s_{1}\right) \\
\vdots \\
\left(\lambda_{i} \pi_{r \chi_{m}^{s c}}{ }^{\left(i+1-\bar{\chi}_{f}^{i-2}\right)}\right)^{1 / 2} \widetilde{A}\left(i, r, s_{1}\right) \\
\left(\lambda_{i} \pi_{r}^{\left(i+1-\bar{\chi}_{f}^{i-2}\right)}\right)^{1 / 2} \widetilde{A}\left(i, r, s_{1}\right) \\
\vdots \\
\left(\lambda_{i} \pi_{r}^{\left(i+1-\bar{\chi}_{f}^{i-2}\right)}\right)^{1 / 2} \widetilde{A}\left(i, r, s_{1}\right)
\end{array}\right] \text {, } \\
& Y(i, r)=\operatorname{diag}\left\{Y_{1}(i, r), Y_{2}(i, r)\right\}, \\
& \widetilde{Y}_{1}(i, r)=\operatorname{diag}\left\{\widetilde{Y}_{1}(i, r), \widetilde{Y}_{2}(i, r)\right\}, \\
& Y_{1}(i, r)=\operatorname{diag}\left\{Y_{1 \chi_{0}^{i-2}}(i, r), \ldots, Y_{1 \chi_{\bar{m}}^{i-2}}(i, r)\right\}, \\
& =\operatorname{diag}\left\{Y_{2 \chi_{\bar{m}+1}^{i-2}}(i, r), \ldots, Y_{2 \chi_{m}^{i-2}}(i, r)\right\}, \\
& \tilde{Y}_{1}(i, r) \\
& =\operatorname{diag}\left\{\widetilde{Y}_{1 \bar{\chi}_{1}^{i-2}}(i, r), \ldots, \widetilde{Y}_{1 \bar{\chi}_{\bar{m}}^{i-2}}(i, r)\right\}, \\
& \tilde{Y}_{2}(i, r) \\
& =\operatorname{diag}\left\{\tilde{Y}_{2 \bar{\chi}_{\bar{m}+1}^{i-2}}(i, r), \ldots, \widetilde{Y}_{2 \bar{\chi}_{\tau^{c c}-m}^{i-2}}(i, r)\right\}, \\
& Y_{1 \chi_{g}^{i-2}}(i, r) \\
& =\operatorname{diag}\left\{Y_{1 \chi_{g}^{i-2} 1}(i, r),\right. \\
& \left.Y_{1 \chi_{g}^{i-2} 2}(i, r), Y_{1 \chi_{g}^{i-2} 3}(i, r)\right\}, \\
& Y_{2 \bar{\chi}_{h}^{i-2}}(i, r) \\
& =\operatorname{diag}\left\{Y_{2 \bar{\chi}_{h}^{i-2} 1}(i, r),\right. \\
& \left.Y_{2 \bar{\chi}_{h}^{i-2} 2}(i, r), Y_{2 \bar{\chi}_{h}^{i-2} 3}(i, r)\right\}, \\
& \widetilde{Y}_{1 \chi_{e}^{i-2}}(i, r) \\
& =\operatorname{diag}\left\{\widetilde{Y}_{1 \chi_{e}^{i-2} 1}(i, r),\right. \\
& \left.\widetilde{Y}_{1 \chi_{e}^{i-2} 2}(i, r), \widetilde{Y}_{1 \chi_{e}^{i-2} 3}(i, r)\right\}, \\
& \tilde{Y}_{2 \bar{\chi}_{f}^{i-2}}(i, r) \\
& =\operatorname{diag}\left\{\widetilde{Y}_{2 \bar{\chi}_{f}^{i-2} 1}(i, r)\right. \text {, } \\
& \left.\widetilde{Y}_{2 \bar{\chi}_{f}^{i-2} 2}(i, r), \widetilde{Y}_{2 \bar{\chi}_{f}^{i-2} 3}(i, r)\right\}, \\
& Y_{1 \chi_{g}^{i-2} s_{1}}(i, r) \\
& =\operatorname{diag}\left\{X\left(\chi_{g}^{i-2}, \chi_{1}^{r}\right), \ldots, X\left(\chi_{g}^{i-2}, \chi_{n}^{r}\right),\right. \\
& \left.X\left(\chi_{g}^{i-2}, \bar{\chi}_{1}^{r}\right), \ldots, X\left(\chi_{g}^{i-2}, \bar{\chi}_{3-n}^{r}\right)\right\}, \\
& Y_{2 \bar{\chi}_{h}^{i-2} s_{1}}(i, r) \\
& =\operatorname{diag}\left\{X\left(\bar{\chi}_{h}^{i-2}, \chi_{1}^{r}\right), \ldots, X\left(\bar{\chi}_{h}^{i-2}, \chi_{n}^{r}\right),\right. \\
& \left.X\left(\bar{\chi}_{h}^{i-2}, \bar{\chi}_{1}^{r}\right), \ldots, X\left(\bar{\chi}_{\tau^{s c}-m}^{i-2}, \bar{\chi}_{3-n}^{r}\right)\right\}, \\
& \tilde{Y}_{1 \chi_{e}^{i-2} s_{1}}(i, r) \\
& =\operatorname{diag}\left\{X\left(\chi_{e}^{i-2}, \chi_{1}^{r}\right), \ldots, X\left(\chi_{e}^{i-2}, \chi_{n}^{r}\right),\right. \\
& \left.X\left(\chi_{e}^{i-2}, \bar{\chi}_{1}^{r}\right), \ldots, X\left(\chi_{e}^{i-2}, \bar{\chi}_{3-n}^{r}\right)\right\},
\end{aligned}
$$




$$
\begin{aligned}
\widetilde{Y}_{2 \bar{\chi}_{f}^{i-2} s_{1}}(i, r) & \\
=\operatorname{diag}\{ & X\left(\bar{\chi}_{f}^{i-2}, \chi_{1}^{r}\right), \ldots, X\left(\bar{\chi}_{f}^{i-2}, \chi_{n}^{r}\right), \\
& \left.X\left(\bar{\chi}_{f}^{i-2}, \bar{\chi}_{1}^{r}\right), \ldots, X\left(\bar{\chi}_{f}^{i-2}, \bar{\chi}_{3-n}^{r}\right)\right\}
\end{aligned}
$$

hold for all $i, j \in \varphi, r, s_{1}, s_{2} \in \phi, \chi_{a}^{r} \in \phi_{\chi}^{r}, \bar{\chi}_{b}^{r} \in \phi_{\mu \chi}^{r}, \chi_{c}^{i} \in$ $\varphi_{\chi}^{i}, \bar{\chi}_{d}^{i} \in \varphi_{\mu \chi}^{i}, \chi_{g}^{i-2} \in \varphi_{\chi}^{i-2}$, and $\bar{\chi}_{h}^{i-2} \in \varphi_{\mu \chi}^{i-2}$, where

$$
\begin{gathered}
\varphi_{\chi}^{i-2}=\left\{\chi_{0}^{i-2}, \chi_{1}^{i-2}, \ldots, \chi_{m}^{i-2}\right\}, \quad 0 \leq \bar{m} \leq m, \\
\bar{\varphi}_{\chi}^{i-2}=\left\{\chi_{\bar{m}+1}^{i-2}, \chi_{\bar{m}+2}^{i-2}, \ldots, \chi_{m}^{i-2}\right\}, \quad 0 \leq m \leq \tau^{s c}, \\
\varphi_{\mu \chi}^{i-2}=\left\{\bar{\chi}_{1}^{i-2}, \bar{\chi}_{2}^{i-2}, \ldots, \bar{\chi}_{\widetilde{m}}^{i-2}\right\}, \quad 1 \leq \widetilde{m} \leq \tau^{s c}-m, \\
\bar{\varphi}_{\mu \chi}^{i-2}=\left\{\bar{\chi}_{\bar{m}^{i-1},}^{i-2} \bar{\chi}_{\bar{m}+2}^{i-2}, \ldots, \bar{\chi}_{\tau^{s c}-m}^{i-2}\right\}, \quad 0 \leq m \leq \tau^{s c}, \\
\pi_{r}^{\left(i+1-\chi_{e}^{i-2}\right)}=1-\sum_{s_{2} \in \phi_{\chi}^{r}} \pi_{r s_{2}}^{\left(i+1-\chi_{e}^{i-2}\right)}, \\
\pi_{r}^{\left(i+1-\bar{\chi}_{f}^{i-2}\right)}=1-\sum_{s_{2} \in \phi_{\chi}^{r}} \pi_{r s_{2}}^{\left(i+1-\bar{\chi}_{f}^{i-2}\right)}, \\
\pi_{r}^{\left(i+1-\chi_{c}^{i}\right)}=1-\sum_{s_{2} \in \phi_{\chi}^{r}} \pi_{r s_{2}}^{\left(i+1-\chi_{c}^{i}\right)}, \\
\pi_{r}^{\left(i+1-\bar{\chi}_{d}^{i}\right)}=1-\sum_{s_{2} \in \phi_{\chi}^{r}} \pi_{r s_{2}}^{\left(i+1-\bar{\chi}_{d}^{i}\right)}, \\
\forall \chi_{e}^{i-2} \in \bar{\varphi}_{\chi}^{i-2}, \bar{\chi}_{f}^{i-2} \in \bar{\varphi}_{\mu \chi}^{i-2}, \chi_{c}^{i} \in \varphi_{\chi}^{i}, \bar{\chi}_{d}^{i} \in \varphi_{\mu \chi}^{i},
\end{gathered}
$$

and $\widetilde{A}\left(i, r, s_{1}\right), \lambda_{i}$, and $\pi_{r}^{(i)}$ are defined in (38).

Proof. By applying the Schur complement and letting $X\left(\chi_{c}^{i}, \chi_{a}^{r}\right)=P^{-1}\left(\chi_{c}^{i}, \chi_{a}^{r}\right), X\left(\bar{\chi}_{d}^{i}, \bar{\chi}_{b}^{r}\right)=P^{-1}\left(\bar{\chi}_{d}^{i}, \bar{\chi}_{b}^{r}\right), X\left(\chi_{c}^{i}, \chi_{a}^{r}\right)=$ $P^{-1}\left(\chi_{c}^{i}, \chi_{a}^{r}\right), X\left(\bar{\chi}_{d}^{i}, \bar{\chi}_{b}^{r}\right)=P^{-1}\left(\bar{\chi}_{d}^{i}, \bar{\chi}_{b}^{r}\right), X\left(\chi_{c}^{i}, \bar{\chi}_{b}^{r}\right)=P^{-1}\left(\chi_{c}^{i}, \bar{\chi}_{b}^{r}\right)$, $X\left(\bar{\chi}_{d}^{i}, \chi_{a}^{r}\right)=P^{-1}\left(\bar{\chi}_{d}^{i}, \chi_{a}^{r}\right), X\left(\chi_{g}^{i-2}, \chi_{a}^{r}\right)=P^{-1}\left(\chi_{g}^{i-2}, \chi_{a}^{r}\right)$, $X\left(\chi_{g}^{i-2}, \bar{\chi}_{b}^{r}\right)=P^{-1}\left(\chi_{g}^{i-2}, \bar{\chi}_{b}^{r}\right), X\left(\chi_{e}^{i-2}, \chi_{a}^{r}\right)=P^{-1}\left(\chi_{e}^{i-2}, \chi_{a}^{r}\right)$, $X\left(\chi_{e}^{i-2}, \bar{\chi}_{b}^{r}\right)=P^{-1}\left(\chi_{e}^{i-2}, \bar{\chi}_{b}^{r}\right), X\left(\bar{\chi}_{f}^{i-2}, \chi_{a}^{r}\right)=P^{-1}\left(\bar{\chi}_{f}^{i-2}, \chi_{a}^{r}\right)$, $X\left(\bar{\chi}_{f}^{i-2}, \bar{\chi}_{b}^{r}\right)=P^{-1}\left(\bar{\chi}_{f}^{i-2}, \bar{\chi}_{b}^{r}\right), X\left(\bar{\chi}_{h}^{i-2}, \bar{\chi}_{b}^{r}\right)=P^{-1}\left(\bar{\chi}_{h}^{i-2}, \bar{\chi}_{b}^{r}\right)$, and $X\left(\bar{\chi}_{h}^{i-2}, \chi_{a}^{r}\right)=P^{-1}\left(\bar{\chi}_{h}^{i-2}, \chi_{a}^{r}\right)$, the proof can be readily completed.

Remark 14 . The nonconvex feasibility problem (45)-(50) can be formulated as an optimisation problem subject to LMI constraints by using Algorithm 10 .

\section{Number Example}

To illustrate the effectiveness of the proposed method, we consider the cart and inverted pendulum problem [8-10] in Figure 2, where $m_{1}$ is the cart mass, $m_{2}$ is the pendulum mass, $L$ is the length from the point of rotation to the center of gravity of the pendulum, $x$ is the cart position, $\theta$ is the

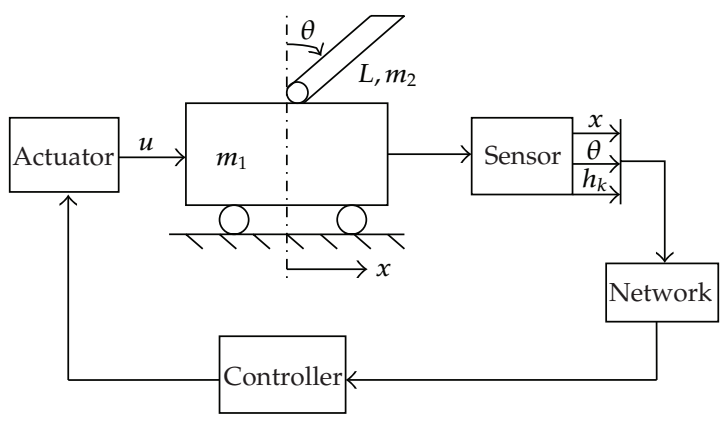

FIGURE 2: Cart and inverted pendulum system.

pendulum angular position, and $u$ is the input force. This is a fourth-order unstable system. The state variables are $x_{1}=$ $x, x_{2}=\dot{x}, x_{3}=\theta$, and $x_{4}=\dot{\theta}$. The parameters used are $m_{1}=1 \mathrm{~kg}, m_{2}=0.5 \mathrm{~kg}$, and $L=1 \mathrm{~m}$ and smooth surfaces. The state-space model is

$$
\dot{x}(t)=A x(t)+B u(t),
$$

where

$$
\begin{gathered}
A=\left[\begin{array}{cccc}
1.0000 & 1.0000 & -0.0005 & 0.0005 \\
0 & 1.0000 & -3.2663 & 0 \\
0 & 0 & -0.0002 & 1.0000 \\
0 & 0 & 19.6007 & -0.0002
\end{array}\right], \\
B=\left[\begin{array}{c}
-0.0003 \\
0.8448 \\
-0.0003 \\
-1.3328
\end{array}\right] .
\end{gathered}
$$

Assume the minimum sampling period is $0.1 s$, the maximum sampling period is $0.3 s$, then $h_{k} \in\{0.1 s, 0.2 s, 0.3 s\}$. Assume the case where random delays only exist from the sensor to the controller, and $\tau_{k}^{s c} \in\{0,1\}$, and their transition probability matrices are given by

$$
\Lambda=\left[\begin{array}{ll}
0.4 & 0.6 \\
0.5 & 0.5
\end{array}\right], \quad \Pi=\left[\begin{array}{lll}
0.5 & 0.4 & 0.1 \\
0.2 & 0.5 & 0.3 \\
0.1 & 0.4 & 0.5
\end{array}\right] \text {. }
$$

Figure 3 shows part of the simulated run of the $S$ - $C$ delays $\tau_{k}^{s c}$ and sampling periods $h_{k}$ governed by their corresponding transition probability matrices, respectively.

In the above assumption, we discretize the system (53) and obtain

$$
\begin{aligned}
\Phi_{1} & =\left[\begin{array}{cccc}
1.1052 & 0.1105 & -0.0177 & -0.0005 \\
0 & 1.1052 & -0.3546 & -0.0172 \\
0 & 0 & 1.0996 & 0.1033 \\
0 & 0 & 2.0247 & 1.0996
\end{array}\right], \\
\Phi_{2} & =\left[\begin{array}{cccc}
1.2214 & 0.2443 & -0.0794 & -0.0049 \\
0 & 1.2214 & -0.8165 & -0.0745 \\
0 & 0 & 1.4182 & 0.2272 \\
0 & 0 & 4.4527 & 1.4182
\end{array}\right],
\end{aligned}
$$



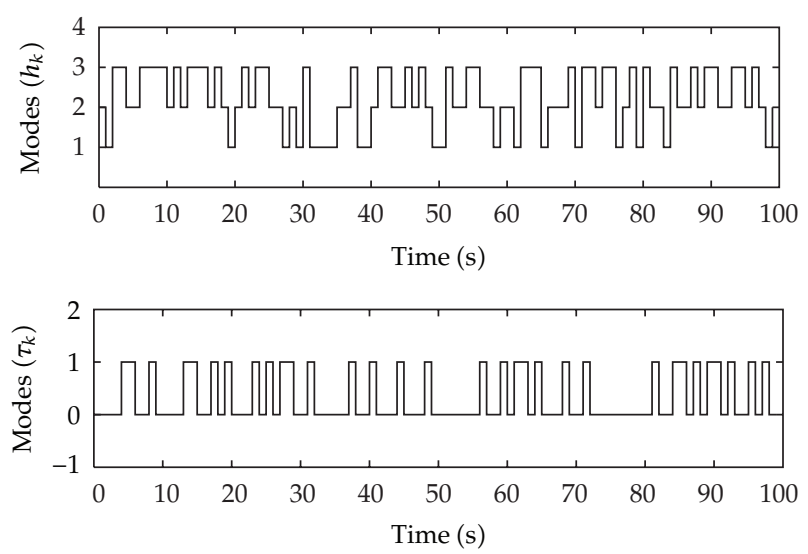

FIGURE 3: Modes with $\tau_{k}$ and $h_{k}$.

$$
\begin{aligned}
\Phi_{3} & =\left[\begin{array}{cccc}
1.3499 & 0.4050 & -0.2055 & -0.0184 \\
0 & 1.3499 & -1.4816 & -0.1872 \\
0 & 0 & 2.0194 & 0.3963 \\
0 & 0 & 7.7676 & 2.0194
\end{array}\right], \\
\Gamma_{1} & =\left[\begin{array}{c}
0.0045 \\
0.0896 \\
-0.0068 \\
-0.1377
\end{array}\right], \quad \Gamma_{2}=\left[\begin{array}{c}
0.0196 \\
0.1934 \\
-0.0285 \\
-0.3029
\end{array}\right], \\
\Gamma_{3} & =\left[\begin{array}{c}
0.0482 \\
0.3187 \\
-0.0694 \\
-0.5285
\end{array}\right] .
\end{aligned}
$$

The eigenvalues of $A$ are $1,1,4.4272$, and -4.4275 , the eigenvalues of $\Phi_{1}$ are $1.1052,1.1052,0.6423$, and 1.5569 , the eigenvalues of $\Phi_{2}$ are $1.2214,1.2214,2.4240$, and 0.4125 , the eigenvalues of $\Phi_{3}$ are 1.3499, 1.3499, 3.7739, and 0.2649. Hence, the system is unstable. The state trajectory of the open-loop system is shown in Figure 4.

By Theorem 8 and Algorithm 10, when transition probabilities are completely known, we can obtain the controllers with the following matrices $K\left(\tau_{k}^{s c}, h_{k-\tau_{k}^{s c}}\right)$ :

$$
\begin{aligned}
& K 01=\left[\begin{array}{llll}
-0.1722 & -1.4488 & 12.0905 & 3.2261
\end{array}\right], \\
& K 02=\left[\begin{array}{llll}
-0.5648 & -1.8662 & 0.8811 & 4.2691
\end{array}\right], \\
& K 03=\left[\begin{array}{llll}
-0.5648 & -1.8662 & 0.8811 & 4.2690
\end{array}\right], \\
& K 11=\left[\begin{array}{llll}
-0.4764 & -1.6341 & 1.1288 & 3.6632
\end{array}\right], \\
& K 12=\left[\begin{array}{llll}
-0.5657 & -1.8687 & 0.8783 & 4.2466
\end{array}\right], \\
& K 13=\left[\begin{array}{llll}
-0.5655 & -1.8397 & 0.8806 & 4.2296
\end{array}\right]
\end{aligned}
$$

By Theorem 13, when transition probabilities are partly known; that is,

$$
\Lambda=\left[\begin{array}{cc}
0.4 & 0.6 \\
? & ?
\end{array}\right], \quad \Pi=\left[\begin{array}{ccc}
0.5 & ? & ? \\
? & 0.5 & ? \\
0.1 & 0.4 & 0.5
\end{array}\right]
$$

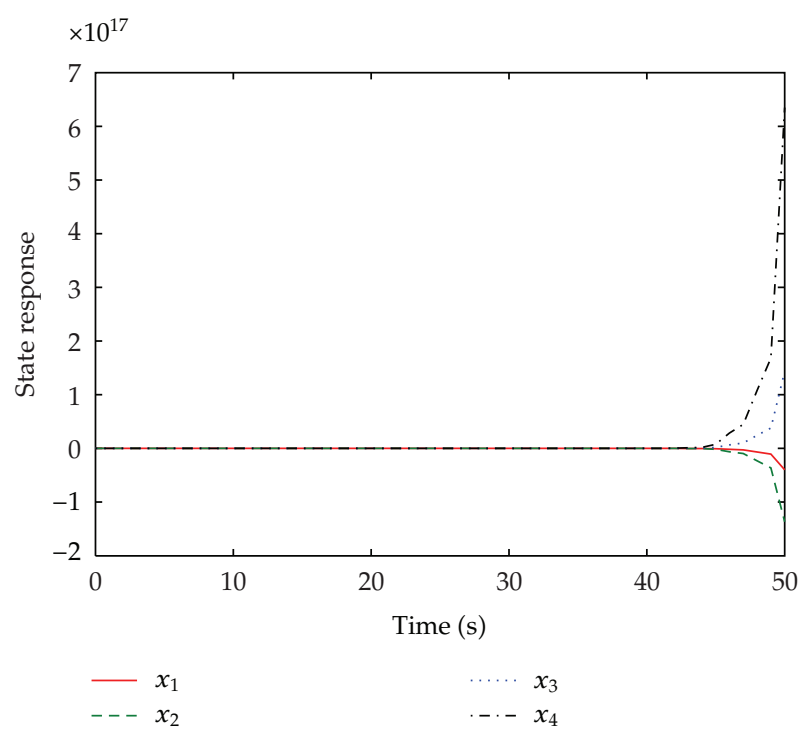

FIgURE 4: States of the open-loop system.

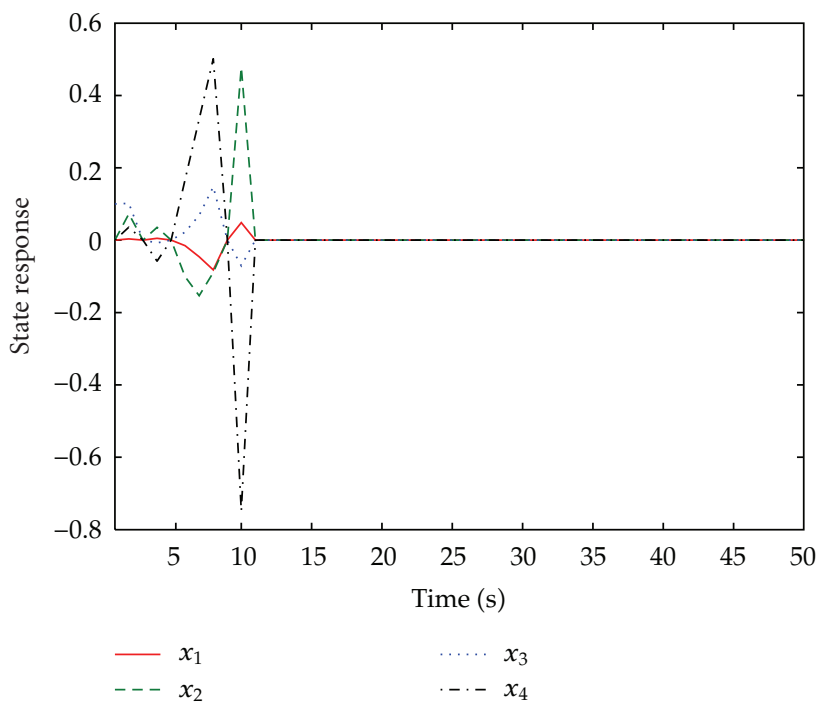

FIGURE 5: States of the closed-loop system (completely known).

we can obtain the controllers with the following matrices $K\left(\tau_{k}^{s c}, h_{k-\tau_{k}^{s c}}\right)$ :

$$
\begin{aligned}
& K 01=\left[\begin{array}{llll}
-0.1534 & 1.5482 & 10.1902 & 2.2463
\end{array}\right], \\
& K 02=\left[\begin{array}{llll}
0.5648 & -1.8662 & -0.5814 & 14.2531
\end{array}\right], \\
& K 03=\left[\begin{array}{llll}
-0.5648 & -1.8662 & 0.8811 & 3.9625
\end{array}\right] \\
& K 11=\left[\begin{array}{llll}
-0.3561 & -1.4026 & 1.4723 & 2.9558
\end{array}\right] \\
& K 12=\left[\begin{array}{llll}
-0.5659 & -1.8432 & 0.8791 & 4.2211
\end{array}\right] \\
& K 13=\left[\begin{array}{llll}
-0.5646 & -1.7649 & 0.8869 & 4.1893
\end{array}\right]
\end{aligned}
$$

Assume that the initial condition for simulation is $\theta(0)=$ $0.1 \mathrm{rad}$, and all other initial states are zero. The state trajectories of the closed-loop system caused by the discretized model are shown in Figures 5 and 6, respectively. 


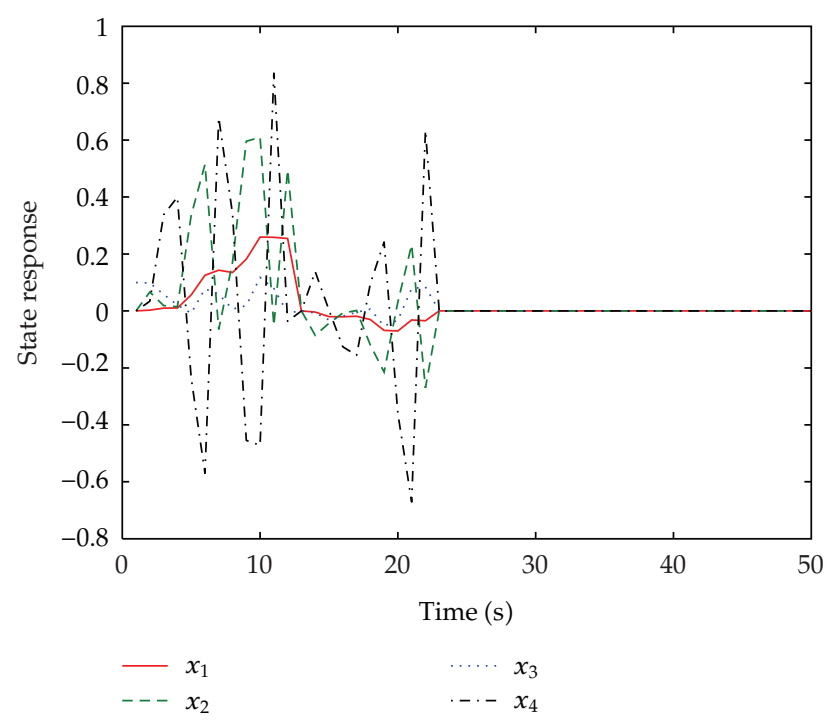

Figure 6: States of the closed-loop system (partly known).

\section{Conclusions}

This paper proposes a state feedback controller design method for NCSs with random time delays and random sampling periods. Sampling periods also can randomly switch between three cases according to the network load. The $S-C$ random delays and random sampling periods are modeled as Markov chains. The transition probabilities do not need to be completely known. The closed-loop systems can be expressed as jump linear systems with two modes. Sufficient conditions of stochastic stability are obtained in terms of a set of LMIs with matrix inversion constraints. Further, the state feedback controller is designed via the iterative linear matrix inequality approach and the state feedback gain depends on the two modes.

\section{Acknowledgments}

This work was supported in part by the Natural Science Foundation of China (NSFC) under Grants nos. 61273008, 61074029, and 61104093.

\section{References}

[1] L. A. Montestruque and P. J. Antsaklis, "On the model-based control of networked systems," Automatica, vol. 39, no. 10, pp. 1837-1843, 2003.

[2] L. A. Montestruque and P. Antsaklis, "Stability of model-based networked control systems with time-varying transmission times," IEEE Transactions on Automatic Control, vol. 49, no. 9, pp. 1562-1572, 2004.

[3] W. Zhang, M. S. Branicky, and S. M. Phillips, "Stability of networked control systems," IEEE Control Systems Magazine, vol. 21, no. 1, pp. 84-97, 2001.

[4] Z. Z. Qiu, Q. L. Zhang, and Z. W. Zhao, "Stability of singular networked control systems with control constraint," Journal of Systems Engineering and Electronics, vol. 18, no. 2, pp. 290-296, 2007.
[5] S. S. Hu and Q. X. Zhu, "Stochastic optimal control and analysis of stability of networked control systems with long delay," Automatica, vol. 39, no. 11, pp. 1877-1884, 2003.

[6] G. C. Walsh, H. Ye, and L. G. Bushnell, "Stability analysis of networked control systems," IEEE Transactions on Control Systems Technology, vol. 10, no. 3, pp. 438-446, 2002.

[7] Y. Wang, Z. C. Ji, L. B. Xie, and W. L. Hu, "Co-design for networked control systems based on vary-period sample approach," Journal of System Simulation, vol. 20, no. 8, pp. 21082114, 2008.

[8] L. Xiao, A. Hassibi, and J. P. How, "Control with random communication delays via a discrete-time jump system approach," in Proceedings of the American Control Conference, vol. 3, pp. 2199-2204, June 2000.

[9] L. Zhang, Y. Shi, T. Chen, and B. Huang, "A new method for stabilization of networked control systems with random delays," IEEE Transactions on Automatic Control, vol. 50, no. 8, pp. 11771181, 2005.

[10] Y. Shi and B. Yu, "Output feedback stabilization of networked control systems with random delays modeled by Markov chains," IEEE Transactions on Automatic Control, vol. 54, no. 7, pp. 1668-1674, 2009.

[11] Y. Shi and B. Yu, "Robust mixed $H_{2} / H_{\infty}$ control of networked control systems with random time delays in both forward and backward communication links," Automatica, vol. 47, no. 4, pp. 754-760, 2011.

[12] D. Huang and S. K. Nguang, "State feedback control of uncertain networked control systems with random time-delays," in Proceedings of the 46th IEEE Conference on Decision and Control (CDC '07), pp. 3964-3969, New Orleans, La, USA, December 2007.

[13] L. X. Zhang and E. K. Boukas, "Stability and stabilization of Markovian jump linear systems with partly unknown transition probabilities," Automatica, vol. 45, no. 2, pp. 463-468, 2009.

[14] L. X. Zhang, E. K. Boukas, and J. Lam, "Analysis and synthesis of Markov Jump Linear systems with time-varying delays and partially known transition probabilities," IEEE Transactions on Automatic Control, vol. 53, no. 10, pp. 2458-2464, 2008.

[15] G. L. Wang, Q. L. Zhang, and V. Sreeram, "Partially modedependent $H_{\infty}$ filtering for discrete-time Markovian jump systems with partly unknown transition probabilities," Signal Processing, vol. 90, no. 2, pp. 548-556, 2010.

[16] Y. G. Sun and S. Y. Qin, "Stability and stabilization of networked control systems with bounded packet dropout," Acta Automatica Sinica, vol. 37, no. 1, pp. 113-117, 2011.

[17] Y. Li, Q. L. Zhang, and Z. Z. Qiu, "Stability of networked control systems with time-varying sampling periods and partially known transition probabilities," International Journal of Information \& Systems Sciences, vol. 7, no. 4, pp. 340-356, 2011.

[18] X. L. Guo, "Controller design based on variable-period sampling approach for Networked Control Systems with random delays," in Proceedings of IEEE International Conference on Networking, Sensing and Control (ICNSC '09), pp. 147-151, March 2009.

[19] Y. L. Wang and G. H. Yang, " $H_{\infty}$ controller design for networked control systems via active-varying sampling period method," Acta Automatica Sinica, vol. 34, no. 7, pp. 814-818, 2008.

[20] Y. Wang, Z. X. Sun, and Z. X., Analysis and Design of Network Control System, Tsinghua University Press, Beijing, China, 2009.

[21] R. X. Wang, Random Process, Xian Jiaotong University Press, Xi'an, China, 2006. 
[22] M. C. de Oliveira and J. C. Geromel, "Numerical comparison of output feedback design methods," in Proceedings of the American Control Conference, vol. 1, pp. 72-76, June 1997.

[23] L. Zhang, B. Huang, and J. Lam, " $H_{\infty}$ model reduction of Markovian jump linear systems," Systems and Control Letters, vol. 50, no. 2, pp. 103-118, 2003.

[24] L. El Ghaoui, F. Oustry, and M. AitRami, "A cone complementarity linearization algorithm for static output-feedback and related problems," IEEE Transactions on Automatic Control, vol. 42, no. 8, pp. 1171-1176, 1997. 


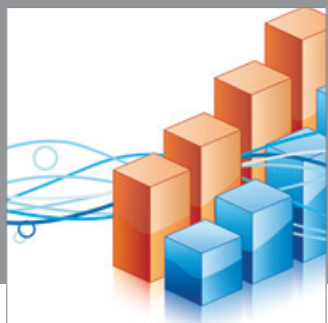

Advances in

Operations Research

mansans

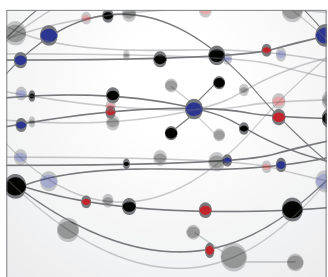

The Scientific World Journal
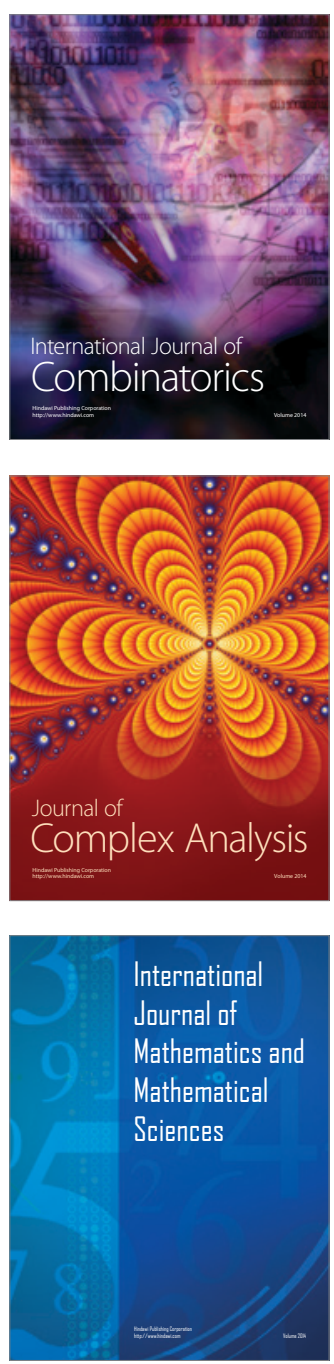
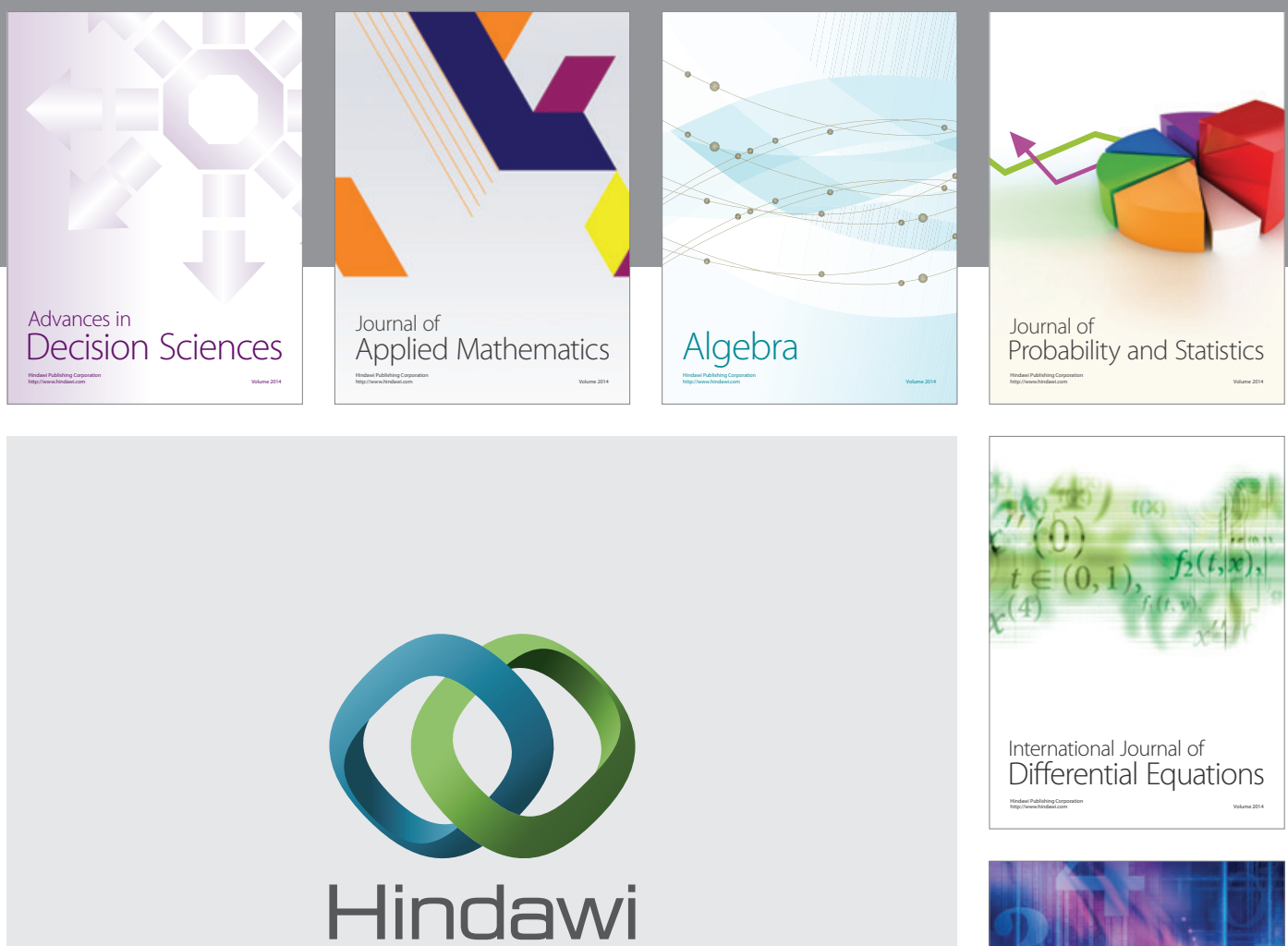

Submit your manuscripts at http://www.hindawi.com
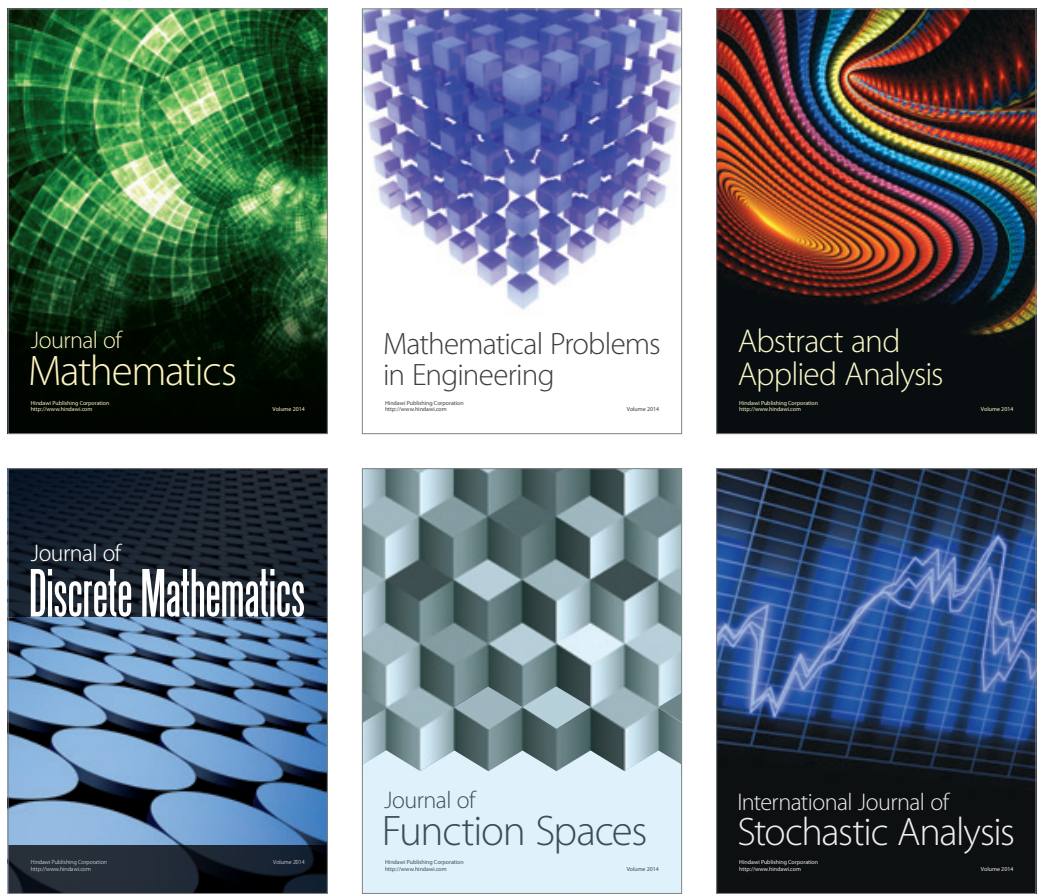

Journal of

Function Spaces

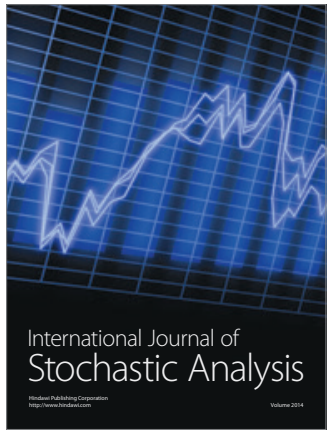

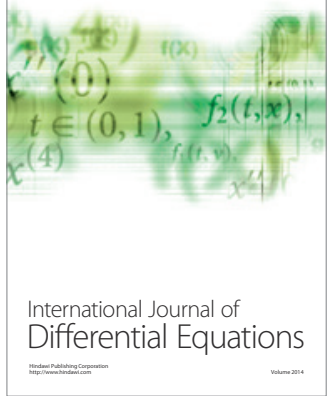
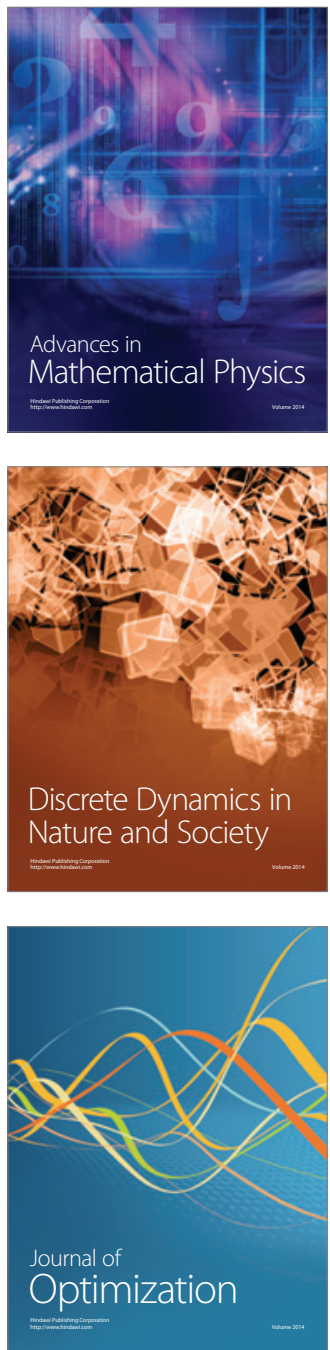